\title{
The Dynamics of a Bouncing Superball With Spin
}

\author{
Philip J Aston \\ R Shail \\ Department of Mathematics \\ University of Surrey \\ Guildford \\ Surrey GU2 7XH \\ U.K.
}

October 11, 2007 


\begin{abstract}
When a superball is thrown forwards but with backspin, it is observed to reverse both direction and spin for a few bounces before settling to bouncing motion in one direction. The bouncing ball is modelled by a two-dimensional iterated map in terms of the horizontal velocity and spin immediately after each bounce. The asymptotic motion of this system is easily determined. However, of more interest is the transient behaviour. The two-dimensional linear map is reduced to a one-dimensional nonlinear map and this map is used to determine the number of direction and spin reversals that can occur for given initial conditions. The model is then generalised to describe a ball bouncing up and down a single step or successively down a staircase.
\end{abstract}

Keywords: Bouncing ball, spin, direction reversal, spin reversal, staircase.

MSC codes: 74H05, 37E30, 37N15 


\section{Introduction}

A number of toys with interesting properties have been studied mathematically. These include the following:

- The tippe top. This simple toy looks a little like an upside-down mushroom. However, when it is spun on a flat surface, it will flip over and stand upright. There are many papers which consider the dynamics of this toy, including $[12,13]$.

- The rattleback. When spun in a particular direction, the rattleback will gradually slow down and then the spinning motion is replaced by oscillating motion. As the oscillations start to slow down, the rattleback then starts spinning in the opposite direction. The modelling of this behaviour has been considered by a number of authors $[3,8,11]$.

- Chaos toys. A number of simple mechanical systems have been shown to demonstrate chaos such as the one described in [1].

In this paper, we consider another very simple toy, namely a superball. The interesting aspects of a superball are that (i) it is highly elastic with a coefficient of restitution close to 1 , and so bounces very high and (ii) at a bounce, it grips the surface very well which can result in a significant change in spin of the ball. These two aspects result in some interesting behaviour for a bouncing superball.

The classical approach to modelling the bounce of a ball is described in texts such as Routh [14]. It is assumed that during the impulsive interaction of the ball with the surface on which it is bouncing, the impulsive friction and normal reaction obey the laws of friction and Routh [14] cites experimental evidence to this effect. However, whilst this approach can describe a single reversal of direction of motion of the ball, it is unable to predict the rich spectrum of back and forward motions which the superball can exhibit.

The modelling of the bounce of a ball in the vertical direction consists of two components, namely (i) a reversal of the vertical velocity and (ii) a reduction in the vertical velocity after the bounce by a (constant) coefficient of restitution. To model the bounce of a superball, the first of these two concepts was extended by Garwin [9] who considered a similar model in the horizontal direction wherein the horizontal motion of the ball at the point of impact is reversed at the bounce. Cross [5] modified Garwin's model by introducing the second component of a horizontal coefficient of restitution that reduces the horizontal velocity of the ball after the bounce by a fixed amount. In both cases, this equation describing the change in the horizontal velocity at the bounce was combined with an equation representing the conservation of angular momentum. These two equations were solved to give the horizontal and angular velocities after the bounce in terms of these quantities before the bounce.

Cross [5] compared the bounce of a superball with the bounce of a tennis ball whereas Garwin [9] was particularly interested in the phenomenon where the superball reverses direction when it is thrown forward to bounce between two horizontal parallel surfaces. This behaviour was also investigated by Hefner [10] but in the case where the parallel surfaces are vertical. He also briefly considered the multiple bounces of a superball on a flat surface.

Bridges [2] used strobe photography to try and measure the horizontal coefficient of restitution under different conditions. The results do not show much uniformity, but it is acknowledged that they are "subject to a higher degree of error than is desirable". Experimental results by Cross [6] show a much more consistent picture for the horizontal coefficient and indicate that it is approximately constant at a value around 0.5 over a wide range of 


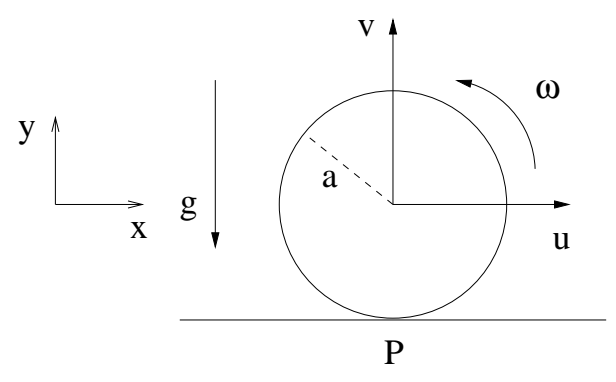

Figure 1: The parameter and variables of the ball.

incident angles, dropping down slightly for small incident angles. Doménech [7] extended the model of Garwin and Cross to three dimensions and also obtained consistent experimental results giving the horizontal coefficient of restitution as 0.5.

In this paper, we consider the bouncing behaviour of a superball with spin on a flat surface in more detail, using the model for the bounce described by Cross [5]. In particular, conditions on the initial conditions for a reversal of direction or spin of the ball are considered. This is extended to finding conditions for $n$ reversals of direction or spin for any $n \geq 1$. We then show that the model requires only a very small change for the case of a ball bouncing up and down a step or successively down a staircase. Several different examples of typical motion are given for this case.

\section{The Model}

\subsection{The model equations}

We consider the motion of a superball of radius $a$ and mass $m$, bouncing on a horizontal plane. The motion is assumed to be two-dimensional and the orientation of the coordinate axes in the vertical plane of motion of the centre, together with the horizontal, vertical and angular velocities, are shown in Fig. $1 . P$ is the point of contact of the ball with the horizontal plane in the bounce. We assume that the bounce is instantaneous and we neglect the geometrical deformation of the ball during the impact as this is effectively taken into account by the coefficient of restitution. During the flight of the ball between bounces any aerodynamic or viscous effects that might arise from the motion of the ball are ignored and so the angular and horizontal velocities remain constant during the flight.

A model of the bounce of a superball was described by Cross [5]. This model was extended by Hefner [10] to include the parabolic trajectory between bounces, and is briefly reviewed here. Let $u_{j}, v_{j}$ and $\omega_{j}$ be the horizontal, vertical and angular velocities of the ball respectively immediately after the $j^{\text {th }}$ bounce. Consider the $(n+1)^{\text {th }}$ bounce; from the elementary mechanics of the parabolic trajectory of the centre of the ball prior to the $(n+1)^{\text {th }}$ bounce it follows that the velocity components just before the $(n+1)^{\text {th }}$ bounce are $u_{n},-v_{n}$ and $\omega_{n}$. If the normal (vertical) coefficient of restitution is $\beta, 0<\beta<1$, then

$$
v_{n+1}=\beta v_{n} .
$$

In the horizontal direction, it is assumed that the horizontal velocity of the ball at the point of contact $P$ is reversed and reduced in magnitude by a tangential (horizontal) coefficient of restitution $\alpha$, where $0<\alpha<1$. This condition is expressed by

$$
u_{n+1}+a \omega_{n+1}=-\alpha\left(u_{n}+a \omega_{n}\right) .
$$


This model supposes that the ball instantly grips the surface at the bounce, deforms elastically and then the motion at the point of contact reverses. What may happen in practice is that the ball slips slightly initially before gripping. Clearly, if this is the case, then the energy put into the reversal of the ball will be reduced. However, this is effectively included in this model in terms of a reduced horizontal coefficient of restitution. Thus, this coefficient is determined by the amount of slipping and the elasticity of the ball.

Note that $\alpha=0$ in (2) corresponds to a model considered by Routh [14] where there is no slipping on impact and no elastic deformation. This case is appropriate for a hard ball which grips the floor but does not permit much of the dynamics that is observed for a superball. The values $\alpha=\beta=1$ are also excluded since they imply that energy is conserved in the bounce, which is unrealistic for a real ball, and because many aspects of the dynamics are different in this conservative case.

A third model equation arises from the conservation of angular momentum in the bounce. In order to avoid introducing the impulsive friction and normal reaction at $P$, we equate angular momenta about $P$ before and after the impact, giving

$$
\frac{2}{5} m a^{2} \omega_{n+1}-m a u_{n+1}=\frac{2}{5} m a^{2} \omega_{n}-\operatorname{mau}_{n} .
$$

From equations (2) and (3) it now follows that

$$
\begin{aligned}
u_{n+1} & =\frac{1}{7}(5-2 \alpha) u_{n}-\frac{2}{7}(1+\alpha) a \omega_{n} \\
\omega_{n+1} & =-\frac{5}{7 a}(1+\alpha) u_{n}+\frac{1}{7}(2-5 \alpha) \omega_{n}
\end{aligned}
$$

These recurrence relations are to be solved, subject to some initial projection data for the motion.

Suppose that the ball is thrown with initial vertical, horizontal and angular velocities $\tilde{v}, \tilde{u}$ and $\tilde{\omega}$ respectively, from a height $\tilde{y}$. The corresponding trajectory of the ball can be followed backwards in time until the ball reaches the floor, which will give the quantities immediately after a non-existent zeroth bounce. These constitute the initial conditions for the iterative relations (1), (4) and (5). Since the horizontal and angular velocities are constant during the flight, then clearly

$$
u_{0}=\tilde{u}, \quad \omega_{0}=\tilde{\omega}
$$

and $v_{0}$ follows from $\tilde{v}$ and $\tilde{y}$.

To determine the full trajectory of the ball, suppose that the $n^{\text {th }}$ bounce takes place at time $t_{n}$ when the horizontal coordinate of the centre of the ball is $x_{n}$. The horizontal velocity $u(t)$ and vertical velocity $v(t)$ during the subsequent flight of the ball are given by

$$
\begin{aligned}
& u(t)=u_{n}, \\
& v(t)=v_{n}-g\left(t-t_{n}\right),
\end{aligned}
$$

and since $y\left(t_{n}\right)=0$, the trajectory after the $n^{\text {th }}$ bounce can be represented parametrically for $t_{n} \leq t \leq t_{n+1}$ as

$$
\begin{aligned}
& y(t)=v_{n}\left(t-t_{n}\right)-\frac{1}{2} g\left(t-t_{n}\right)^{2}, \\
& x(t)=x_{n}+u_{n}\left(t-t_{n}\right),
\end{aligned}
$$

where $(x(t), y(t))$ is the position of the ball centre at time $t$. 
Two further results follow trivially, namely that the time and position of the $(n+1)^{\text {th }}$ bounce are given in terms of the corresponding quantities for the $n^{\text {th }}$ bounce by

$$
\begin{aligned}
t_{n+1} & =t_{n}+\frac{2 v_{n}}{g} \\
x_{n+1} & =x_{n}+\frac{2 u_{n} v_{n}}{g} .
\end{aligned}
$$

\subsection{Non-dimensionalisation and solution}

Equations (1), (4) and (5) are non-dimensionalised with respect to $v_{0}$, which must be nonzero for the ball to bounce at all. Thus, we define non-dimensional variables $V_{n}, U_{n}$ and $\Omega_{n}$ by

$$
V_{n}=\frac{v_{n}}{v_{0}}, \quad U_{n}=\frac{u_{n}}{v_{0}}, \quad \Omega_{n}=\frac{a \omega_{n}}{v_{0}} .
$$

In terms of these new variables, equations (1), (4) and (5) become

$$
\begin{aligned}
V_{n+1} & =\beta V_{n} \\
U_{n+1} & =\frac{1}{7}(5-2 \alpha) U_{n}-\frac{2}{7}(1+\alpha) \Omega_{n} \\
\Omega_{n+1} & =-\frac{5}{7}(1+\alpha) U_{n}+\frac{1}{7}(2-5 \alpha) \Omega_{n} .
\end{aligned}
$$

The initial conditions for this iteration are

$$
V_{0}=1, \quad U_{0}=\frac{u_{0}}{v_{0}}, \quad \Omega_{0}=\frac{a \omega_{0}}{v_{0}}
$$

We note that the six parameters $a, \alpha, \beta, u_{0}, v_{0}$ and $\omega_{0}$ in the original equations have now been reduced to the four non-dimensional parameters $\alpha, \beta, u_{0} / v_{0}, a \omega_{0} / v_{0}$ in these equations.

Clearly equation (11) decouples from equations (12) and (13), and the solution of this first equation is given by

$$
V_{n}=\beta^{n}
$$

since $V_{0}=1$. Thus, we concentrate on (12) and (13), which can be written in matrix form as

$$
\left(\begin{array}{c}
U_{n+1} \\
\Omega_{n+1}
\end{array}\right)=\left(\begin{array}{cc}
\frac{1}{7}(5-2 \alpha) & -\frac{2}{7}(1+\alpha) \\
-\frac{5}{7}(1+\alpha) & \frac{1}{7}(2-5 \alpha)
\end{array}\right)\left(\begin{array}{c}
U_{n} \\
\Omega_{n}
\end{array}\right)=A\left(\begin{array}{c}
U_{n} \\
\Omega_{n}
\end{array}\right), \text { say. }
$$

It can be proved by induction that

$$
A^{n}=\left(\begin{array}{cc}
\frac{1}{7}\left(5+2(-\alpha)^{n}\right) & -\frac{2}{7}\left(1-(-\alpha)^{n}\right) \\
-\frac{5}{7}\left(1-(-\alpha)^{n}\right) & \frac{1}{7}\left(2+5(-\alpha)^{n}\right)
\end{array}\right)
$$

and hence the solution of equation (16) is given by

$$
\begin{aligned}
U_{n} & =\frac{1}{7}\left(5+2(-\alpha)^{n}\right) U_{0}-\frac{2}{7}\left(1-(-\alpha)^{n}\right) \Omega_{0}, \\
\Omega_{n} & =-\frac{5}{7}\left(1-(-\alpha)^{n}\right) U_{0}+\frac{1}{7}\left(2+5(-\alpha)^{n}\right) \Omega_{0},
\end{aligned}
$$


as first found (in dimensional coordinates) by Hefner [10]. We note from these solutions that as $n \rightarrow \infty$,

$$
U_{\infty}=\frac{1}{7}\left(5 U_{0}-2 \Omega_{0}\right), \quad \Omega_{\infty}=-\frac{1}{7}\left(5 U_{0}-2 \Omega_{0}\right),
$$

for all $U_{0}$ and $\Omega_{0}$. Note that if $U_{\infty} \neq 0$, then the bouncing motion of the ball is followed by the ball rolling along the floor with initial velocity $U_{\infty}$.

It also follows from (17) and (18) that

$$
\lim _{n \rightarrow \infty} \frac{U_{n}}{\Omega_{n}}=\left\{\begin{aligned}
-1 & \text { if } U_{0} \neq \frac{2}{5} \Omega_{0}, \\
\frac{2}{5} & \text { if } U_{0}=\frac{2}{5} \Omega_{0} .
\end{aligned}\right.
$$

The additional equations (9) and (10) can also be non-dimensionalised by defining the non-dimensional variables

$$
X_{n}=\frac{g x_{n}}{v_{0}^{2}}, \quad T_{n}=\frac{g t_{n}}{v_{0}} .
$$

In terms of these non-dimensional quantities, equations (9) and (10) become

$$
\begin{aligned}
T_{n+1} & =T_{n}+2 V_{n}, \\
X_{n+1} & =X_{n}+2 U_{n} V_{n} .
\end{aligned}
$$

These equations can be used to calculate the total time, $T_{\text {tot }}$, measured from the first bounce at $T_{1}$ to $T_{\infty}, X_{n}$ and the final horizontal positional coordinate, $X_{\infty}$, of the ball. The results are

$$
\begin{aligned}
T_{\text {tot }} & =\frac{2 \beta}{1-\beta} \\
X_{n} & =X_{1}+\frac{2}{7}\left(\frac{\beta\left(1-\beta^{n-1}\right)}{1-\beta}\left(5 U_{0}-2 \Omega_{0}\right)-\frac{2 \alpha \beta\left(1-(-\alpha \beta)^{n-1}\right)}{1+\alpha \beta}\left(U_{0}+\Omega_{0}\right)\right), \\
X_{\infty} & =X_{1}+\frac{2 \beta}{7}\left(\frac{5 U_{0}-2 \Omega_{0}}{1-\beta}-\frac{2 \alpha\left(U_{0}+\Omega_{0}\right)}{1+\alpha \beta}\right) .
\end{aligned}
$$

In terms of the original coordinates, the total time taken is given by

$$
t_{\mathrm{tot}}=\frac{2 \beta v_{0}}{g(1-\beta)}
$$

and the final position of the ball is given by

$$
x_{\infty}=x_{1}+\frac{2 \beta v_{0}}{7 g}\left(\frac{5 u_{0}-2 a \omega_{0}}{1-\beta}-\frac{2 \alpha\left(u_{0}+a \omega_{0}\right)}{1+\alpha \beta}\right) .
$$

We note that both the total time and the displacement $x_{\infty}-x_{1}$ are both finite and are directly proportional to $v_{0}$ and that the total time is independent of $u_{0}$ and $\omega_{0}$.

\section{Eigenmodes}

The eigenvalues of the matrix $A$ are $\lambda_{1}=1$ and $\lambda_{2}=-\alpha$, with corresponding eigenvectors $\mathbf{x}_{1}=(1,-1)^{T}$ and $\mathbf{x}_{2}=(2 / 5,1)^{T}$. Since the eigenvalues are distinct, the eigenvectors form a basis for $\mathbf{R}^{2}$, and the initial conditions can be written as

$$
\left(\begin{array}{c}
U_{0} \\
\Omega_{0}
\end{array}\right)=c_{1} \mathbf{x}_{1}+c_{2} \mathbf{x}_{2}
$$


for some constants $c_{1}$ and $c_{2}$. It follows from this that

$$
\left(\begin{array}{c}
U_{n} \\
\Omega_{n}
\end{array}\right)=c_{1} \lambda_{1}^{n} \mathbf{x}_{1}+c_{2} \lambda_{2}^{n} \mathbf{x}_{2}
$$

Substituting for the eigenvalues gives

$$
\left(\begin{array}{c}
U_{n} \\
\Omega_{n}
\end{array}\right)=c_{1} \mathbf{x}_{1}+c_{2}(-\alpha)^{n} \mathbf{x}_{2}
$$

The solutions given in (17) and (18) can be identified with this eigenvector basis as they can be rewritten as

$$
\left(\begin{array}{c}
U_{n} \\
\Omega_{n}
\end{array}\right)=\frac{1}{7}\left(5 U_{0}-2 \Omega_{0}\right)\left(\begin{array}{r}
1 \\
-1
\end{array}\right)+\frac{5}{7}\left(U_{0}+\Omega_{0}\right)(-\alpha)^{n}\left(\begin{array}{c}
2 / 5 \\
1
\end{array}\right),
$$

giving

$$
c_{1}=\frac{1}{7}\left(5 U_{0}-2 \Omega_{0}\right), \quad c_{2}=\frac{5}{7}\left(U_{0}+\Omega_{0}\right) .
$$

Thus, all solutions are a linear combination of the two eigenmodes associated with either $c_{1}=0$ or $c_{2}=0$, and to understand all the possible solutions it is sufficient to understand the eigenmodes.

\subsection{First eigenmode $\left(\lambda_{1}=1\right)$}

If $c_{2}=0$, then $c_{1} \mathbf{x}_{1}=\left(U_{0}, \Omega_{0}\right)^{T}$ which implies that $U_{0}=-\Omega_{0}$. In this case,

$$
\left(\begin{array}{c}
U_{n} \\
\Omega_{n}
\end{array}\right)=\left(\begin{array}{c}
U_{0} \\
\Omega_{0}
\end{array}\right) \text { for all } n
$$

and so $U_{n}=-\Omega_{n}$ for all $n$ also.

This motion corresponds to the case when the horizontal velocity of the lowest point $P$ of the ball at impact is zero at each bounce and hence must be zero during the flight also. This is essentially a rolling motion in the horizontal direction, combined with the up and down motion between bounces in the vertical direction associated with the parabolic trajectory. We refer to this as the rolling mode.

\subsection{Second eigenmode $\left(\lambda_{2}=-\alpha\right)$}

If $c_{1}=0$, then $c_{2} \mathbf{x}_{2}=\left(U_{0}, \Omega_{0}\right)^{T}$ which implies that $U_{0}=\frac{2}{5} \Omega_{0}$. In this case,

$$
\left(\begin{array}{c}
U_{n} \\
\Omega_{n}
\end{array}\right)=(-\alpha)^{n}\left(\begin{array}{c}
U_{0} \\
\Omega_{0}
\end{array}\right)
$$

and also $U_{n}=\frac{2}{5} \Omega_{n}$ for all $n$. Clearly, this motion has a change of sign for both $U_{n}$ and $\Omega_{n}$ at each iteration, corresponding to a change of horizontal direction and spin at each bounce.

This motion can be understood more easily by considering $X_{n}$, the horizontal position of the $n^{\text {th }}$ bounce. Combining $(24),(25)$ and using $U_{0}=\frac{2}{5} \Omega_{0}$ gives

$$
X_{n}-X_{\infty}=-\frac{2(-\alpha \beta)^{n}}{1+\alpha \beta} U_{0} .
$$


Since $\alpha \beta>0$, the difference $X_{n}-X_{\infty}$ alternates in sign at every iteration, which implies that the bounces of the ball alternate either side of $X_{\infty}$ while converging to $X_{\infty}$ as $n \rightarrow \infty$ since $\alpha \beta<1$. We refer to this as the oscillating mode.

When $\alpha=0$, as considered by Routh [14], the second eigenvalue is also zero and so, after the first bounce of the ball, the motion consists of only the single rolling mode. This explains why this model cannot exhibit all the dynamics observed for a superball.

The two eigenmodes form a basis for all motions of the ball, since all other cases are a linear combination of these two fundamental modes, and provide a useful framework for understanding the asymptotic behaviour of the solutions. However, the transient motion involves a combination of the rolling and oscillating modes and may result in a finite number of direction or spin reversals, a situation which we explore in more detail in the next section.

\section{Direction and Spin Reversal}

A bouncing superball with spin can be observed to reverse both its (horizontal) direction and spin a number of times before settling down to a more regular bouncing motion in one direction. We consider what we can derive from the model regarding these reversals.

At the $n^{\text {th }}$ bounce there will be a change in the horizontal direction of motion of the ball if $U_{n+1} U_{n}<0$. Substituting for $U_{n+1}$ from (12) this becomes

$$
\left(\frac{1}{7}(5-2 \alpha) U_{n}-\frac{2}{7}(1+\alpha) \Omega_{n}\right) U_{n}<0 .
$$

A necessary condition for this to hold is that $U_{n} \neq 0$ and so we divide by $2(1+\alpha) U_{n}^{2}>0$ to give

$$
\frac{\Omega_{n}}{U_{n}}>\frac{5-2 \alpha}{2(1+\alpha)} .
$$

Note that since $0<\alpha<1,(5-2 \alpha) / 2(1+\alpha)>0$. Thus, we can rewrite condition (28) for reversal of direction of the ball as

$$
0<\frac{U_{n}}{\Omega_{n}}<\frac{2(1+\alpha)}{5-2 \alpha} .
$$

The left-hand inequality occurs as there is an implicit requirement in (28) that $\Omega_{n} / U_{n}$ is finite.

Similarly, there will be a change in spin of the ball if $\Omega_{n+1} \Omega_{n}<0$. Substituting for $\Omega_{n+1}$ from (13) this condition becomes

$$
\left(\frac{5}{7}(1+\alpha) U_{n}-\frac{1}{7}(2-5 \alpha) \Omega_{n}\right) \Omega_{n}<0 .
$$

A necessary condition for this to hold is that $\Omega_{n} \neq 0$ and so we divide by $5(1+\alpha) \Omega_{n}^{2}>0$ to give the condition for reversal of spin of the ball as

$$
\frac{2-5 \alpha}{5(1+\alpha)}<\frac{U_{n}}{\Omega_{n}}
$$

Combining (29) and (30), we conclude that there will be a reversal in both direction and spin if

$$
\max \left(0, \frac{2-5 \alpha}{5(1+\alpha)}\right)<\frac{U_{n}}{\Omega_{n}}<\frac{2(1+\alpha)}{5-2 \alpha} .
$$




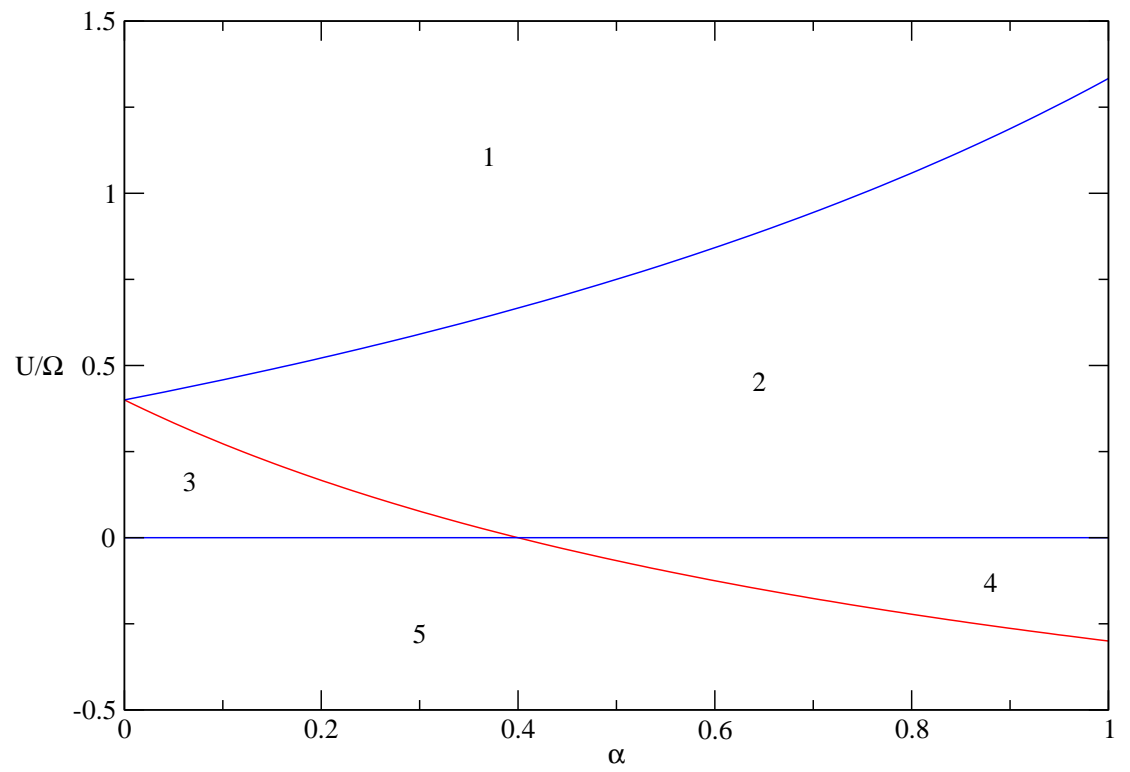

Figure 2: The boundaries on $\frac{U}{\Omega}$ as a function of $\alpha$ for reversals. Upper and lower boundaries for direction reversal (blue); lower boundary for spin reversal (red).

The boundaries for direction and spin reversal as a function of $\alpha$ are shown in Fig. 2 and these boundaries divide the strip $0<\alpha<1,-\infty<U_{n} / \Omega_{n}<\infty$ into five regions. Writing $p=2(1+\alpha) /(5-2 \alpha)$ and $q=(2-5 \alpha) / 5(1+\alpha)$, we have:

1. $p<U_{n} / \Omega_{n}<\infty$ : spin reversal only;

2. $\max (0, q)<U_{n} / \Omega_{n}<p$ : spin and direction reversal;

3. $0<U_{n} / \Omega_{n}<q$ with $\alpha<\frac{2}{5}$ : direction reversal only;

4. $q<U_{n} / \Omega_{n}<0$ with $\alpha>\frac{2}{5}$ : spin reversal only;

5. $-\infty<U_{n} / \Omega_{n}<\min (0, q)$ : neither spin nor direction reversal.

It is worth noting that if $\alpha>2 / 5$ then every direction reversal is accompanied by a spin reversal, but if $\alpha<2 / 5$ then in region 3 there is reversal of direction but not of spin.

\section{The Number of Reversals}

We now consider how many bounces will involve a change of direction or spin for given initial values of $U_{0}$ and $\Omega_{0}$. We start by observing that the previous conditions on reversal of direction and spin involved only the single quantity $\tau_{n}=U_{n} / \Omega_{n}$. Moreover, the dynamics of this quantity is determined by a one-dimensional map, found by dividing (12) by (13) to give

$$
\tau_{n+1}=\frac{U_{n+1}}{\Omega_{n+1}}=\frac{(5-2 \alpha) U_{n}-2(1+\alpha) \Omega_{n}}{-5(1+\alpha) U_{n}+(2-5 \alpha) \Omega_{n}}=\frac{(5-2 \alpha) \tau_{n}-2(1+\alpha)}{-5(1+\alpha) \tau_{n}+(2-5 \alpha)} .
$$

(32) can be regarded as a bilinear mapping of a (real) one-dimensional projective line to itself with $\tau$ as a projective parameter. 


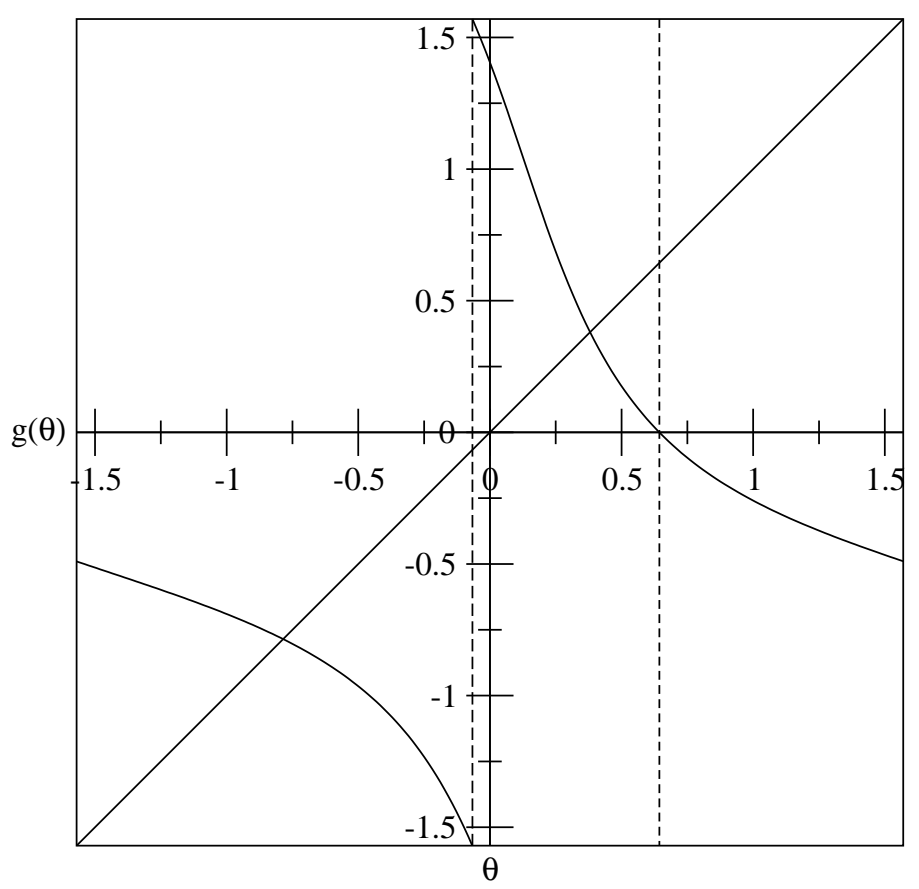

Figure 3: The function $g(\theta)$ for $\alpha=0.5$. Also shown is the line $y=x$, the upper boundary in $\theta$ for direction reversal ( - _ - ) and the lower boundary in $\theta$ for spin reversal ( $\left.-{ }_{-}\right)$. The two fixed points can be seen where the curve $g(\theta)$ intercepts the line $y=x$.

\section{$5.1 \quad \theta$-dynamics}

The problem with working with the variable $\tau_{n}$ in (32) is that it is defined on the whole of the real line, and as $\Omega_{n} \rightarrow 0, \tau_{n} \rightarrow \pm \infty$. A more convenient form for this one-dimensional map can be obtained by transforming the coordinates $U_{n}$ and $\Omega_{n}$ to polar coordinates $\left(R_{n}, \theta_{n}\right)$ :

$$
U_{n}=R_{n} \sin \theta_{n}, \quad \Omega_{n}=R_{n} \cos \theta_{n} .
$$

In these coordinates, $\tau_{n}=\tan \theta_{n}$ and, in terms of $\theta_{n}$, the map is

$$
\theta_{n+1}=g\left(\theta_{n}\right)=\tan ^{-1}\left(\frac{(5-2 \alpha) \tan \theta_{n}-2(1+\alpha)}{-5(1+\alpha) \tan \theta_{n}+2-5 \alpha}\right) .
$$

The function $g(\theta),-\pi / 2<\theta \leq \pi / 2$, is shown in Fig. 3 for $\alpha=0.5$.

Some remarks about (32) and (34) are appropriate. Since $\tan \theta$ has period $\pi,(34)$ is periodic for $\theta_{n} \in\left(-\frac{\pi}{2}, \frac{\pi}{2}\right]$; also, all inequalities involving $U / \Omega$ are preserved when transformed to $\theta \operatorname{since} \tan \theta$ is a monotonically increasing function. Further, the two maps (32) and (34) are topologically conjugate since they are related by the invertible transformation $\tau_{n}=$ $\tan \theta_{n}$. Thus, the dynamics of the two maps is essentially the same as the iterates are related by this invertible coordinate transformation. This means that we can work with either map and we often find it convenient to work with the map (32). It is also apparent that the iterates $\theta_{n}$ are well defined for all $n$ except in the degenerate case of $U_{0}=\Omega_{0}=0$, and that since the linear equations (16) are invertible and the transformation function $U_{n} / \Omega_{n}=\tan \theta_{n}$ is also invertible, then $g$ must also be invertible. 
In terms of $\theta_{n}$, the condition (29) for direction reversal at a bounce is given by

$$
0<\theta_{n}<\tan ^{-1}\left(\frac{2(1+\alpha)}{5-2 \alpha}\right) .
$$

Similarly, the condition (30) for spin reversal is

$$
\tan ^{-1}\left(\frac{2-5 \alpha}{5(1+\alpha)}\right)<\theta_{n}<\frac{\pi}{2}
$$

The right-hand inequality arises from the implicit assumption that $U_{n} / \Omega_{n}$ in (30) is finite.

If $\left(U_{n}, \Omega_{n}\right)^{T}$ is chosen to be an eigenvector of the matrix $A$, then

$$
\left(\begin{array}{c}
U_{n+1} \\
\Omega_{n+1}
\end{array}\right)=\lambda\left(\begin{array}{c}
U_{n} \\
\Omega_{n}
\end{array}\right)
$$

where $\lambda$ is the corresponding eigenvalue. In terms of the polar coordinates defined by (33), this eigenvector case corresponds to

$$
R_{n+1}=\lambda R_{n}, \quad \theta_{n+1}=\theta_{n}
$$

Thus, eigenvectors are associated with fixed points of the one-dimensional map (34).

\section{Lemma 5.1}

The map (34) has two fixed points which are given by

$$
\theta_{1}^{*}=\tan ^{-1}(-1)=-\frac{\pi}{4} \text { and } \theta_{2}^{*}=\tan ^{-1}\left(\frac{2}{5}\right)=0.380506
$$

Moreover, $\theta_{1}^{*}$ is stable and $\theta_{2}^{*}$ is unstable for all values of $\alpha$ and $\lim _{n \rightarrow \infty} \theta_{n}=\theta_{1}^{*}$ if $\theta_{0} \neq \theta_{2}^{*}$.

\section{Proof}

Fixed points of $g$ are associated with the two eigenvectors of the matrix $A$. For the eigenvector corresponding to the eigenvalue $\lambda_{1}=1$, we have $U_{n} / \Omega_{n}=-1$ for all $n$; thus, there is a fixed point of $g$ at $\theta_{1}^{*}=\tan ^{-1}(-1)=-\frac{\pi}{4}$. For the eigenvector corresponding to the eigenvalue $\lambda_{2}=-\alpha$, we have $U_{n} / \Omega_{n}=\frac{2}{5}$ for all $n$ and so there is a fixed point of $g$ at $\theta_{2}^{*}=\tan ^{-1}(2 / 5)=0.380506$. Clearly these fixed points can also be found by solving the equation $\theta=g(\theta)$ or (32) with $\tau_{n+1}=\tau_{n}=\tau$, say, but the above approach highlights the relationship between the fixed points of $g$ and the eigenvectors of $A$. Further, the fixed points of $g$ are independent of $\alpha$ since the eigenvectors of $A$ are themselves independent of $\alpha$.

To determine the stability of these fixed points, we require $g^{\prime}\left(\theta_{1,2}^{*}\right)$. Now from $(34)$, an elementary calculation shows that

$$
g^{\prime}(\theta)=\frac{-49 \alpha \sec ^{2} \theta}{\{-5(1+\alpha) \tan \theta+(2-5 \alpha)\}^{2} \sec ^{2}(g(\theta))} .
$$

If $\theta^{*}$ is a fixed point of $g$, then $g\left(\theta^{*}\right)=\theta^{*}$, whence

$$
g^{\prime}\left(\theta^{*}\right)=\frac{-49 \alpha}{\left\{-5(1+\alpha) \tan \theta^{*}+(2-5 \alpha)\right\}^{2}} .
$$


Evaluating this derivative for the two fixed points of $g$ given in (37), we find that

$$
g^{\prime}\left(\theta_{1}^{*}\right)=-\alpha \quad \text { and } \quad g^{\prime}\left(\theta_{2}^{*}\right)=-\frac{1}{\alpha}
$$

Since $0<\alpha<1$, we conclude that the fixed point $\theta_{1}^{*}=-\frac{\pi}{4}$ is stable for all values of $\alpha$ while the other fixed point $\theta_{2}^{*}=\tan ^{-1}\left(\frac{2}{5}\right)$ is unstable for all values of $\alpha$. It also follows from the asymptotic behaviour $(20)$ that if $\theta_{0} \neq \theta_{2}^{*}$, then $\lim _{n \rightarrow \infty} \theta_{n}=\theta_{1}^{*}$.

In Lemma 5.1, we have established that the function $g$ has two fixed points and that iterates converge to $\theta_{1}^{*}$ for all initial conditions $\theta_{0} \neq \theta_{2}^{*}$. We now consider the way that the iterates converge to $\theta_{1}^{*}$. To do this, we note that $\theta$ is a periodic variable with period $\pi$ and so we consider the phase space for $\theta$ to be a circle of circumference $\pi$. On this circle, there are the two fixed points given in Lemma 5.1. We divide the circle (excluding the fixed points) into the two parts $I_{1}=\left(\theta_{1}^{*}, \theta_{2}^{*}\right)$ and $I_{2}=\left(\theta_{2}^{*}, \theta_{1}^{*}\right)$.

\section{Theorem 5.2}

(i) $g\left(I_{1}\right)=I_{2}$ and $g\left(I_{2}\right)=I_{1}$ and so if $\theta_{n} \in I_{1}\left(I_{2}\right)$, then $\theta_{n+1} \in I_{2}\left(I_{1}\right)$.

(ii) The intervals $I_{1}$ and $I_{2}$ are invariant under $g^{2}$, i.e. $g^{2}\left(I_{1}\right)=I_{1}$ and $g^{2}\left(I_{2}\right)=I_{2}$.

(iii) If $\theta_{0} \neq \theta_{2}^{*}$, then the even and the odd iterates of $\theta_{n}$ converge monotonically on the circle to $\theta_{1}^{*}$.

\section{Proof}

(i) Rather than taking the interval for the $\pi$-periodic function $\theta$ as $\left(-\frac{\pi}{2}, \frac{\pi}{2}\right]$, we take instead the interval $\left[\theta_{2}^{*}, \theta_{2}^{*}+\pi\right)=\left\{\theta_{2}^{*}\right\} \cup I_{2} \cup\left\{\theta_{1}^{*}\right\} \cup I_{1}$. The function $g$ on this interval is shown in Fig. 4 for $\alpha=0.5$. Now

$$
\theta_{n+1}-\theta_{1}^{*}=g\left(\theta_{n}\right)-g\left(\theta_{1}^{*}\right)=g^{\prime}\left(c_{n}\right)\left(\theta_{n}-\theta_{1}^{*}\right),
$$

by the Mean Value Theorem for some $c_{n}$ between $\theta_{n}$ and $\theta_{1}^{*}$. It can be seen from (38) that $g^{\prime}(\theta)<0$ for all values of $\theta$ and so, in particular, $g^{\prime}\left(c_{n}\right)<0$, whatever the value of $c_{n}$ may be. It thus follows from (39) that

$$
\left(\theta_{n+1}-\theta_{1}^{*}\right)\left(\theta_{n}-\theta_{1}^{*}\right)<0
$$

and hence that

$$
g\left(I_{1}\right)=I_{2} \quad \text { and } \quad g\left(I_{2}\right)=I_{1}
$$

(ii) This follows immediately from (i).

(iii) The intervals $I_{1}$ and $I_{2}$ will each contain either the even or the odd iterates since these intervals are invariant under $g^{2}$ by (ii). Since $g$ is invertible and the iterates converge to $\theta_{1}^{*}$ as $n \rightarrow \infty$ from Lemma 5.1 , then the result follows.

The dynamics of $\theta$ on the circle which is described in Lemma 5.1 and Theorem 5.2 is illustrated in Fig. 5.

We note that $\theta_{2}^{*}$ corresponds to a fixed point of (32) given by $\frac{U_{2}^{*}}{\Omega_{2}^{*}}=\frac{2}{5}$. This unstable fixed point is contained in the interval where there is reversal of both direction and spin 


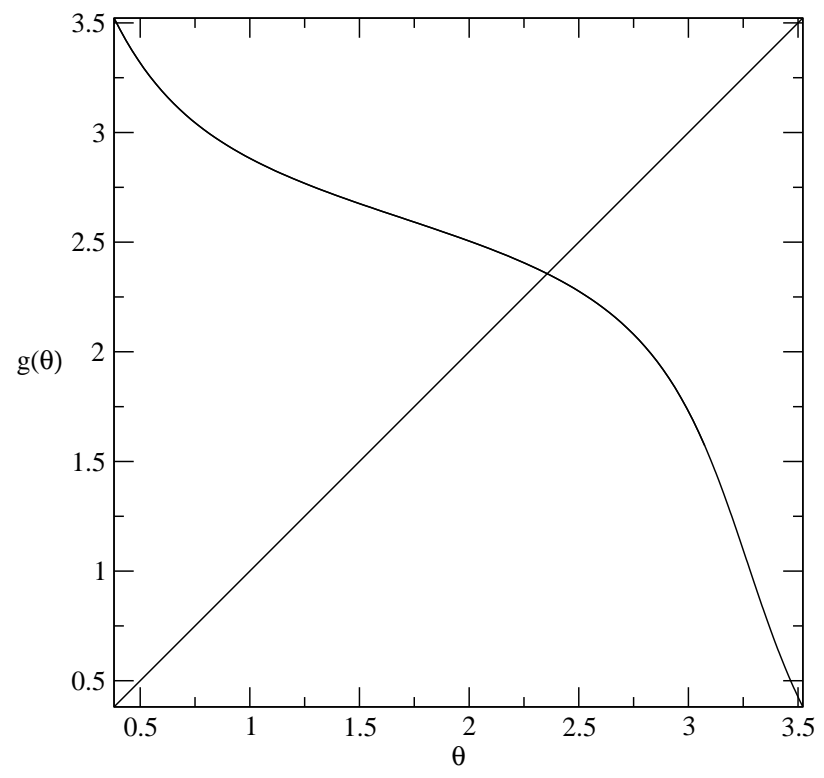

Figure 4: The function $g$ defined on the interval $\left[\theta_{2}^{*}, \theta_{2}^{*}+\pi\right)$ for $\alpha=0.5$.

given by (31) for all $0<\alpha<1$. To see this, we note that the upper and lower boundaries in (31) both converge to $\frac{2}{5}$ as $\alpha \rightarrow 0$. Moreover, it is easy to see that the upper boundary is monotonically increasing and the lower boundary is monotonically decreasing as $\alpha$ increases. This can also be seen from the plot of these boundaries shown in Fig. 2. Thus, the fixed point must always lie between these boundaries.

\subsection{Direction reversals}

From (35) the condition for a direction reversal at the $n^{\text {th }}$ bounce is $\theta_{l}<\theta_{n}<\theta_{r}$, where $\theta_{l}=0$ and $\theta_{r}=\tan ^{-1}\{2(1+\alpha) /(5-2 \alpha)\}$ are the left- and right-hand points of the interval. Define $I_{d}=\left(\theta_{\ell}, \theta_{r}\right)$, the interval associated with direction reversals; then from $(34)$,

$$
g\left(\theta_{r}\right)=\theta_{\ell},
$$

that is $\theta_{r}$ corresponds to the back iterate of the left-hand boundary point $\theta_{l}$.

We now back-iterate $\theta_{r}$, so that

$$
g\left(\theta_{\ell}^{1}\right)=\theta_{r}
$$

it is found that $\theta_{\ell}^{1}=\tan ^{-1}\left\{2\left(1-\alpha^{2}\right) /\left(5+2 \alpha^{2}\right)\right\}$. If $\theta_{0} \in\left(\theta_{\ell}, \theta_{\ell}^{1}\right)$, then $\theta_{1}=g\left(\theta_{0}\right) \notin I_{d}$. Thus, there is only one direction reversal in this case. Similarly, we can back-iterate $\theta_{\ell}^{1}$, so that

$$
g\left(\theta_{r}^{1}\right)=\theta_{\ell}^{1}
$$

with $\theta_{r}^{1}=\tan ^{-1}\left\{2\left(1+\alpha^{3}\right) /\left(5-2 \alpha^{3}\right)\right\}$. Now if $\theta_{0} \in\left(\theta_{r}^{1}, \theta_{r}\right)$, then $\theta_{1} \in\left(\theta_{\ell}, \theta_{\ell}^{1}\right)$ and so $\theta_{2} \notin I_{d}$. In this case, there are two direction reversals. By continuing this process of back iteration, successive intervals can be found which are associated with a particular number of direction reversals.

As the iterates of $g$ oscillate either side of $\theta_{2}^{*}$ by Theorem 5.2, then clearly the back iterates will oscillate in a similar way. Also, as $\theta_{2}^{*}$ is an unstable fixed point for $g$ (see Lemma 5.1), 


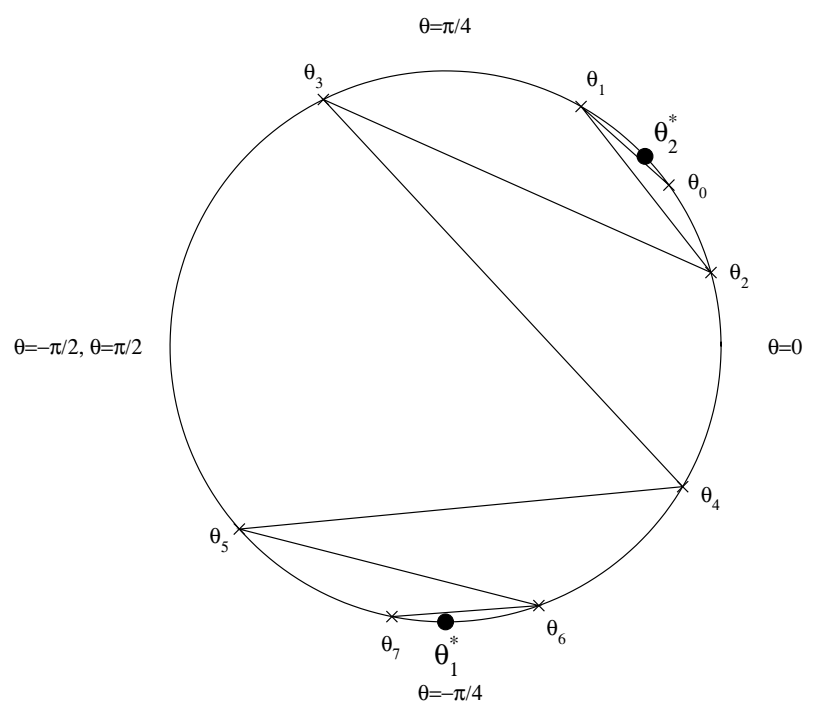

Figure 5: The dynamics on the circle.

then it must be a stable fixed point for $g^{-1}$, and so the back iterates will converge towards $\theta_{2}^{*}$ as $n \rightarrow \infty$. Clearly the size of the interval associated with $n$ reversals of direction will reduce as $n$ increases.

To calculate the boundaries of the intervals associated with a particular number of direction reversals, it is more convenient to work with the two linear equations (16) rather than the single nonlinear equation (34). Inverting the matrix $A$, the inverse of the map (16) is given by

$$
\left(\begin{array}{c}
U_{n} \\
\Omega_{n}
\end{array}\right)=-\frac{1}{7 \alpha}\left(\begin{array}{cc}
2-5 \alpha & 2(1+\alpha) \\
5(1+\alpha) & 5-2 \alpha
\end{array}\right)\left(\begin{array}{c}
U_{n+1} \\
\Omega_{n+1}
\end{array}\right)=B\left(\begin{array}{c}
U_{n+1} \\
\Omega_{n+1}
\end{array}\right) \text {, say. }
$$

We prefer to write this map as a forward iteration in terms of the new variables $\tilde{U}_{n}$ and $\tilde{\Omega}_{n}$ :

$$
\left(\begin{array}{c}
\tilde{U}_{n+1} \\
\tilde{\Omega}_{n+1}
\end{array}\right)=B\left(\begin{array}{c}
\tilde{U}_{n} \\
\tilde{\Omega}_{n}
\end{array}\right)
$$

It is easily shown that, for integer $m$,

$$
B^{m}=\frac{(-1)^{m}}{7 \alpha^{m}}\left(\begin{array}{cc}
2+5(-\alpha)^{m} & 2\left(1-(-\alpha)^{m}\right) \\
5\left(1-(-\alpha)^{m}\right) & 5+2(-\alpha)^{m}
\end{array}\right),
$$

and, using (43) with $m=2 n$, it follows that

$$
\frac{\tilde{U}_{2 n}}{\tilde{\Omega}_{2 n}}=\frac{\left(2+5 \alpha^{2 n}\right) \tilde{U}_{0}+2\left(1-\alpha^{2 n}\right) \tilde{\Omega}_{0}}{5\left(1-\alpha^{2 n}\right) \tilde{U}_{0}+\left(5+2 \alpha^{2 n}\right) \tilde{\Omega}_{0}} .
$$

Setting $\tilde{U}_{0}=0$ gives

$$
\frac{\tilde{U}_{2 n}}{\tilde{\Omega}_{2 n}}=\frac{2\left(1-\alpha^{2 n}\right)}{5+2 \alpha^{2 n}} .
$$


For $n=1,2, \ldots,(45)$ gives the boundary points to the left of the fixed point $\tau^{*}=2 / 5$ of (32) (this is an unstable fixed point of (32) and hence a stable fixed point of the inverse of (32)). Thus, we conclude that if

$$
\frac{2\left(1-\alpha^{2 n-2}\right)}{5+2 \alpha^{2 n-2}}<\frac{U_{0}}{\Omega_{0}}<\frac{2\left(1-\alpha^{2 n}\right)}{5+2 \alpha^{2 n}}
$$

then there will be $2 n-1$ direction reversals, $n=1,2, \ldots$

Similarly, from (43) with $m=2 n-1$, it follows that

$$
\frac{\tilde{U}_{2 n-1}}{\tilde{\Omega}_{2 n-1}}=\frac{\left(2-5 \alpha^{2 n-1}\right) \tilde{U}_{0}+2\left(1+\alpha^{2 n-1}\right) \tilde{\Omega}_{0}}{5\left(1+\alpha^{2 n-1}\right) \tilde{U}_{0}+\left(5-2 \alpha^{2 n-1}\right) \tilde{\Omega}_{0}} .
$$

Setting $\tilde{U}_{0}=0$ then gives

$$
\frac{\tilde{U}_{2 n-1}}{\tilde{\Omega}_{2 n-1}}=\frac{2\left(1+\alpha^{2 n-1}\right)}{5-2 \alpha^{2 n-1}}
$$

and, by an argument similar to that leading to (46), there will be $2 n$ direction reversals, $n=1,2, \ldots$, if

$$
\frac{2\left(1+\alpha^{2 n+1}\right)}{5-2 \alpha^{2 n+1}}<\frac{U_{0}}{\Omega_{0}}<\frac{2\left(1+\alpha^{2 n-1}\right)}{5-2 \alpha^{2 n-1}}
$$

The boundaries for the different regions of direction reversals are shown in Fig. 6 .

It is easily seen from (46) and (48) that if $0<U_{0} / \Omega_{0}<2 / 5$, then there will be an odd number of direction reversals of the ball, whereas if $2 / 5<U_{0} / \Omega_{0}<2(1+\alpha) /(5-2 \alpha)$, then there will be an even number of direction reversals of the ball. The 'permanance' of the motion after the cessation of direction reversals is illuminated by the following result.

\section{Theorem 5.3}

For the bouncing ball, once direction reversals have ended, they cannot start again.

\section{Proof}

In the description of the $\theta$-dynamics earlier in this section, we noted that there is a particular interval in $I_{d}$, the interval associated with direction reversals, where the next iterate escapes from $I_{d}$. To be precise, if $\theta_{n} \in\left(\theta_{\ell}, \theta_{\ell}^{1}\right) \subset I_{d}$, then $\theta_{n+1} \notin I_{d}$, where $\theta_{\ell}$ and $\theta_{\ell}^{1}$ are defined in (40) and (41) respectively. In this case, it follows that $\theta_{n+1} \in\left(\theta_{r}, g^{2}\left(\theta_{r}\right)\right)$.

Continuing to iterate, we note that $\theta_{n+2} \in\left(g^{2}\left(\theta_{\ell}^{1}\right), \theta_{\ell}\right)$. This interval must have no intersection with $I_{d}$ since the iterates of $g^{2}$ move monotonically away from $\theta_{2}^{*}$ and towards $\theta_{1}^{*}$ by Theorem 5.2. All subsequent iterates will move further from $\theta_{2}^{*}$ and towards $\theta_{1}^{*}$ and so they will never enter $I_{d}$ again. Thus, once the iterates escape from $I_{d}$ they will never return, which proves the stated result.

It follows from the results of this section that if the number of direction reversals is even, then the ball will settle down to motion in the same direction as the initial velocity $U_{0}$, whereas, if the number of direction reversals is odd, then the ball will settle down to motion in the opposite direction to the initial velocity $U_{0}$.

\subsection{Spin reversals}

The analysis of spin reversals is similar to that for direction reversals. For $n=1,2, \ldots$, the boundary between there being a reversal of spin and no reversal occurs at the point $\Omega_{n}=0$, 


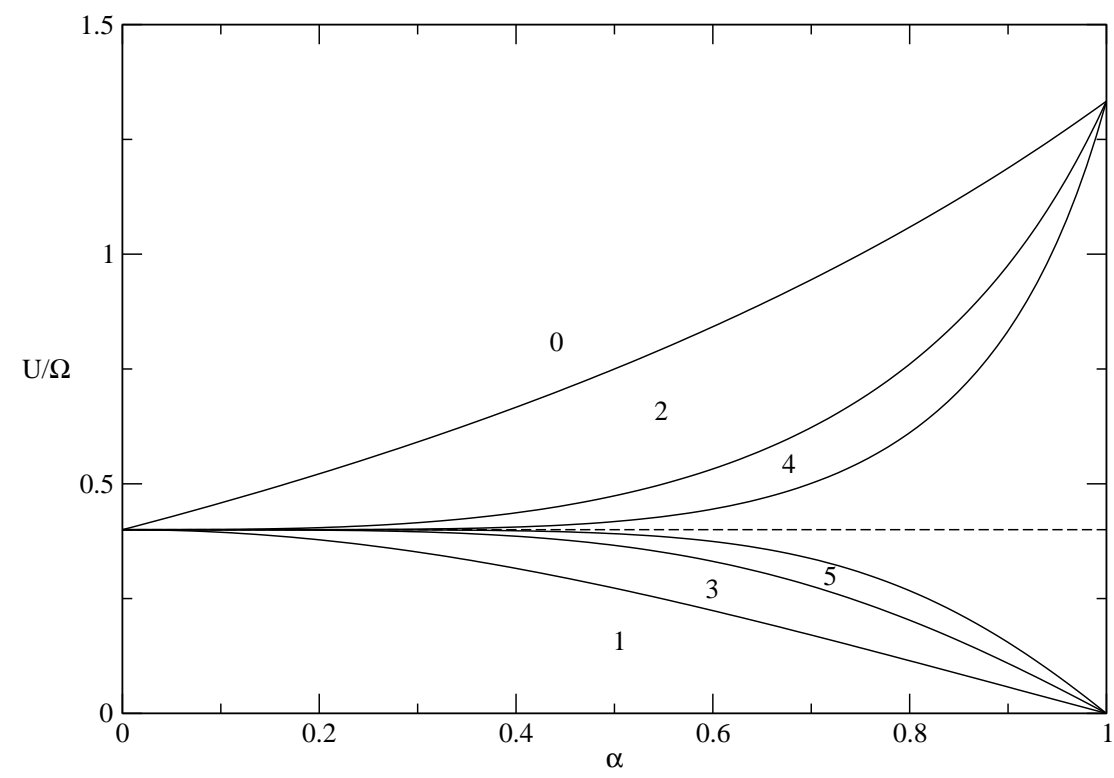

Figure 6: The boundaries which define regions associated with different numbers of direction reversals. The fixed point at $\frac{U}{\Omega}=\frac{2}{5}$ is also shown $(----)$.

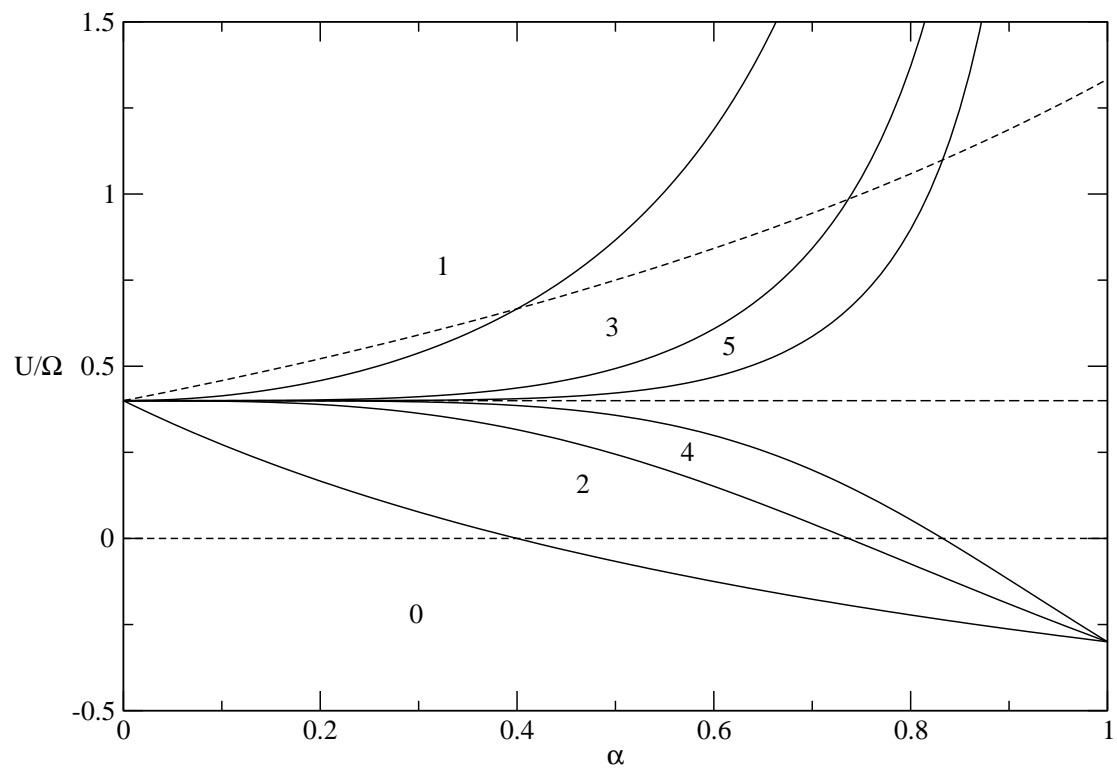

Figure 7: The boundaries which define regions associated with different numbers of spin reversals. The fixed point at $\frac{U}{\Omega}=\frac{2}{5}$ is also shown $(----)$, as are the upper and lower boundaries for direction reversal (- - - - - ). 


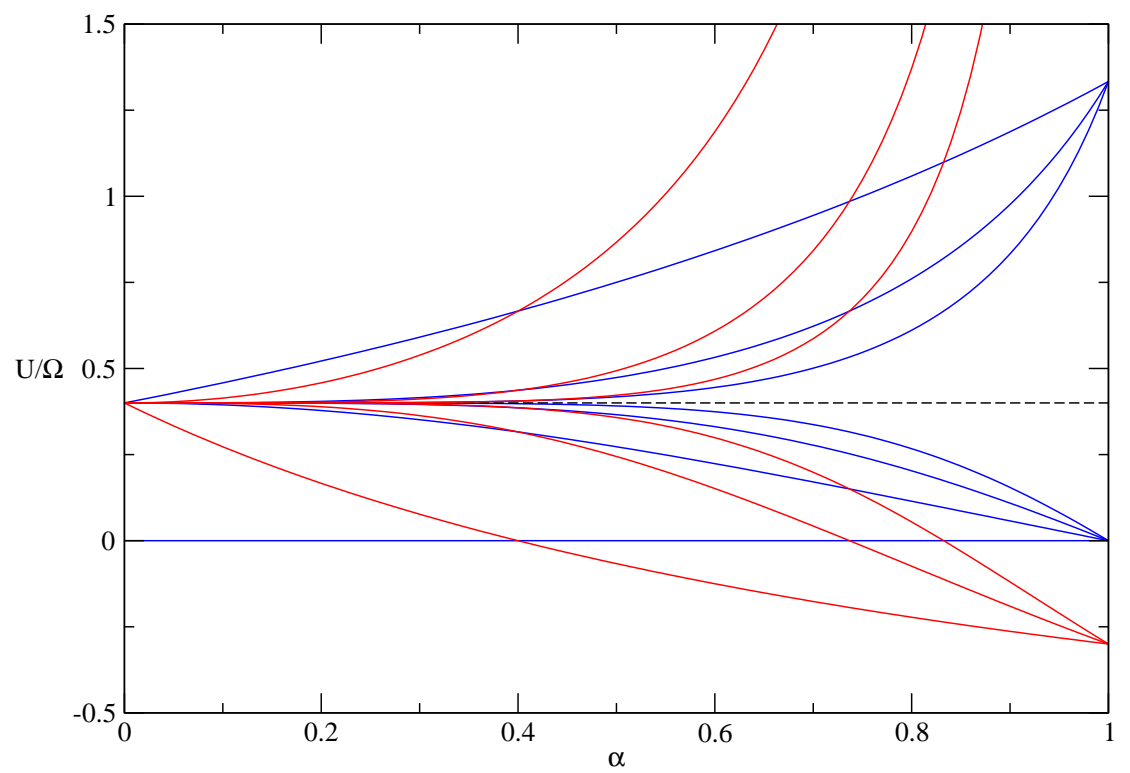

Figure 8: The boundaries which define regions associated with different numbers of direction reversals (blue) and spin reversals (red). The fixed point at $\frac{U}{\Omega}=\frac{2}{5}$ is also shown $(----)$.

which corresponds to $\theta_{n}=\frac{\pi}{2}$. To find intervals associated with a particular number of spin reversals, we back iterate this point. These iterates will again oscillate either side of the fixed point $\theta_{2}^{*}$ and will converge to this fixed point as $n \rightarrow \infty$, resulting in successively smaller intervals.

Setting $\tilde{\Omega}_{0}=0$ in (44) and following the arguments of the previous section, it is found that if

$$
\frac{2+5 \alpha^{2 n}}{5\left(1-\alpha^{2 n}\right)}<\frac{U_{0}}{\Omega_{0}}<\frac{2+5 \alpha^{2 n-2}}{5\left(1-\alpha^{2 n-2}\right)},
$$

then there are $2 n-1$ spin reversals, $n=2,3, \ldots$. We note that when $n=1$, the term on the right-hand side of this inequality involves a division by zero, reflecting the fact that it corresponds to the point $\Omega_{0}=0$. Thus, for $n=1$, there is only one condition, namely

$$
\frac{2+5 \alpha^{2}}{5\left(1-\alpha^{2}\right)}<\frac{U_{0}}{\Omega_{0}}
$$

and this range results in a single reversal of spin. Similarly, setting $\tilde{\Omega}_{0}=0$ in (47) gives the condition that if

$$
\frac{2-5 \alpha^{2 n-1}}{5\left(1+\alpha^{2 n-1}\right)}<\frac{U_{0}}{\Omega_{0}}<\frac{2-5 \alpha^{2 n+1}}{5\left(1+\alpha^{2 n+1}\right)},
$$

then there will be $2 n$ spin reversals, $n=1,2, \ldots$ It follows from (49) and (51) that if $(2-5 \alpha) / 5(1+\alpha)<U_{0} / \Omega_{0}<2 / 5$, there will be an even number of spin reversals of the ball, whereas if $2 / 5<U_{0} / \Omega_{0}$, there will be an odd number of spin reversals.

The boundaries for the different regions of spin reversals are shown in Fig. 7, together with the boundaries of the region where there is direction reversal. Fig. 8 shows the boundaries for direction and spin reversal superimposed. The condition under which there are spin reversals without direction reversal is provided by the following lemma: 


\section{Lemma 5.4}

For $\alpha>(2 / 5)^{1 /(2 n-1)}$, there are values of $U_{0} / \Omega_{0}$ where there are $2 n-1$ or $2 n$ reversals of spin but no direction reversals.

\section{Proof}

From Fig. 7 it can be seen that the regions for different numbers of spin reversals move outside of the direction reversal area for sufficiently large $\alpha$. The boundary for $2 n$ spin reversals crosses the lower boundary for direction reversals when

$$
\frac{2-5 \alpha^{2 n-1}}{5\left(1+\alpha^{2 n-1}\right)}=0
$$

that is when $\alpha=(2 / 5)^{1 /(2 n-1)}$. Similarly, the boundary for $2 n-1$ spin reversals crosses the upper boundary for direction reversals when

$$
\frac{2+5 \alpha^{2 n}}{5\left(1-\alpha^{2 n}\right)}=\frac{2(1+\alpha)}{5-2 \alpha}
$$

and this equation also simplifies to $2-5 \alpha^{2 n-1}=0$, that is $\alpha=(2 / 5)^{1 /(2 n-1)}$.

Using a method analogous to that of Theorem 5.3, it is easily shown that once spin reversals have ended, they cannot start again. If the number of spin reversals is even, then the ball will settle down to having spin in the same direction as the initial spin $\Omega_{0}$ whereas, if the number of spin reversals is odd, then the ball will settle down to having spin in the opposite direction to the initial spin $\Omega_{0}$. We further note that if $U_{0} / \Omega_{0}$ is changed so that its value crosses one of the boundaries we have defined relating to reversal of either direction or spin, then there will be an increase or decrease of two in the number of reversals. This is due to the oscillating nature of the solutions arising from the second eigenmode. See the examples in Section 7 to observe this effect.

\section{The Special Case $\alpha=2 / 5$}

When $\alpha=2 / 5$, there is a strong correlation between reversals of direction and spin. To see this, we note that in this case the boundaries for direction reversal are given by

$$
\frac{\tilde{U}_{2 n-1}^{d}}{\tilde{\Omega}_{2 n-1}^{d}}=\frac{2\left[1+\left(\frac{2}{5}\right)^{2 n-1}\right]}{5-2\left(\frac{2}{5}\right)^{2 n-1}}, \quad \frac{\tilde{U}_{2 n}^{d}}{\tilde{\Omega}_{2 n}^{d}}=\frac{2\left[1-\left(\frac{2}{5}\right)^{2 n}\right]}{5+2\left(\frac{2}{5}\right)^{2 n}},
$$

where the superscript $d$ denotes direction reversal. Similarly, the boundaries for spin reversal are supplied by

$$
\frac{\tilde{U}_{2 n-1}^{s}}{\tilde{\Omega}_{2 n-1}^{s}}=\frac{2-5\left(\frac{2}{5}\right)^{2 n-1}}{5\left[1+\left(\frac{2}{5}\right)^{2 n-1}\right]}, \quad \frac{\tilde{U}_{2 n}^{s}}{\tilde{\Omega}_{2 n}^{s}}=\frac{2+5\left(\frac{2}{5}\right)^{2 n}}{5\left[1-\left(\frac{2}{5}\right)^{2 n}\right]} .
$$

Plainly,

$$
\frac{\tilde{U}_{2 n-1}^{d}}{\tilde{\Omega}_{2 n-1}^{d}}=\frac{\tilde{U}_{2 n}^{s}}{\tilde{\Omega}_{2 n}^{s}} \text { and } \frac{\tilde{U}_{2 n}^{d}}{\tilde{\Omega}_{2 n}^{d}}=\frac{\tilde{U}_{2 n-1}^{s}}{\tilde{\Omega}_{2 n-1}^{s}} .
$$

Thus, in this special case, the boundaries of spin and direction reversal coincide. A number of results follow from this observation. 


\section{Theorem 6.1}

If $\alpha=2 / 5$, then

(i) there will be one reversal of spin and no direction reversals if $U_{0} / \Omega_{0}>2 / 3$;

(ii) there will be $2 n$ reversals of spin and $2 n-1$ direction reversals, $n=1,2, \ldots$, if

$$
\frac{2\left(1-\left(\frac{2}{5}\right)^{2 n-2}\right)}{5+2\left(\frac{2}{5}\right)^{2 n-2}}<\frac{U_{0}}{\Omega_{0}}<\frac{2\left(1-\left(\frac{2}{5}\right)^{2 n}\right)}{5+2\left(\frac{2}{5}\right)^{2 n}} ;
$$

(iii) there will be $2 n+1$ reversals of spin and $2 n$ direction reversals, $n=1,2, \ldots$ if

$$
\frac{2\left(1+\left(\frac{2}{5}\right)^{2 n+1}\right)}{5-2\left(\frac{2}{5}\right)^{2 n+1}}<\frac{U_{0}}{\Omega_{0}}<\frac{2\left(1+\left(\frac{2}{5}\right)^{2 n-1}\right)}{5-2\left(\frac{2}{5}\right)^{2 n-1}} ;
$$

(iv) the number of reversals of spin is one more than the number of direction reversals;

(v) once reversals have finished (assuming that $U_{0} / \Omega_{0} \neq 2 / 5$ ), either the direction or the spin will be in a different direction from the initial direction, but not both.

\section{Proof}

(i) This result follows immediately by setting $\alpha=2 / 5$ in (29) and (50).

(ii) This follows by setting $\alpha=2 / 5$ in (46) and (51), noting the first relation in (52).

(iii) Setting $\alpha=2 / 5$ in (48) and (49), and noting the second relation in (52) gives (iii).

(iv) This is immediate from (i) through (iii).

(v) This result follows from the previous one as an even or odd number of reversals imply that the final direction is the same as or opposite to the inital direction.

\section{Examples}

To illustrate these results, we take the two coefficients of restitution to be $\beta=0.9$ and $\alpha=0.5$, which are close to the experimental values obtained by Cross [6]. The initial values of $U_{0}$ and $\Omega_{0}$ can now be chosen to give different numbers of direction and spin reversals. Taking $U_{0}=0.36$ and $\Omega_{0}=1$ gives $U_{0} / \Omega_{0}=0.36$, which corresponds to 3 reversals of direction and 6 reversals of spin, whilst choosing $U_{0}=0.38$ gives $U_{0} / \Omega_{0}=0.38$, corresponding to 5 reversals of direction but still 6 reversals of spin. The results in these two cases are shown in Figs 9 and 10. We note from (19) that when $U_{0} / \Omega_{0}=0.36$, the limiting values for $U_{n}$ and $\Omega_{n}$ as $n \rightarrow \infty$ are given by $U_{\infty}=-\Omega_{\infty}=-0.02857$ and are $U_{\infty}=-\Omega_{\infty}=-0.01429$ when $U_{0} / \Omega_{0}=0.38$.

In Figs $9(\mathrm{a})$ and 10(a), the movement of the $\theta_{n}$-iterates away from the fixed point $\theta_{2}^{*}$ and towards the fixed point $\theta_{1}^{*}$ can be seen. In addition, in Figs $9(\mathrm{~b})$ and $10(\mathrm{~b})$, the monotonic convergence of the even and odd $\theta_{n}$-iterates to the fixed point $\theta_{1}^{*}$ is evident. 


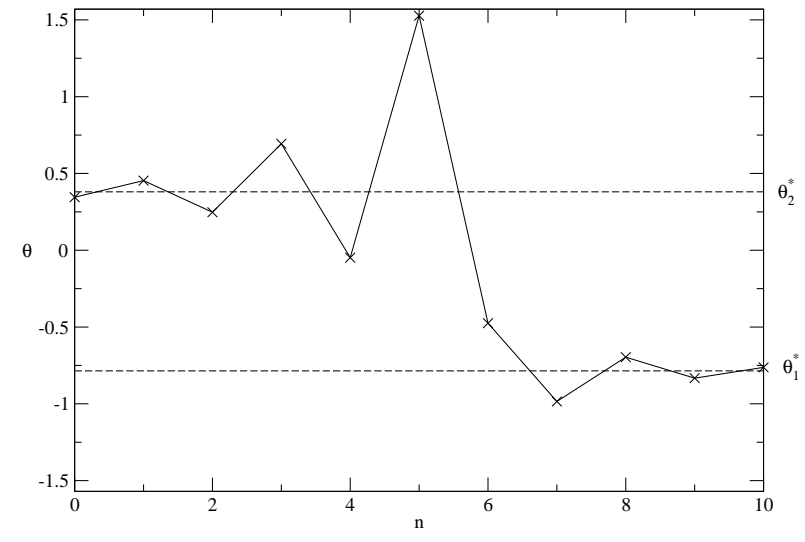

(c)

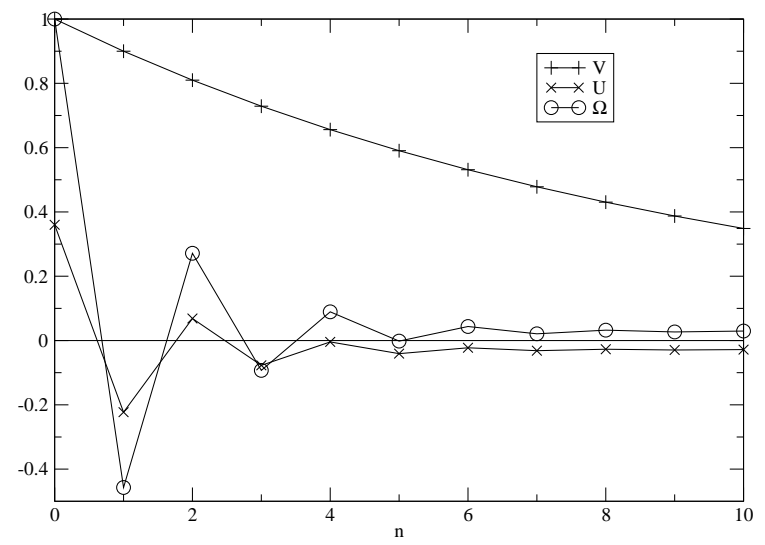

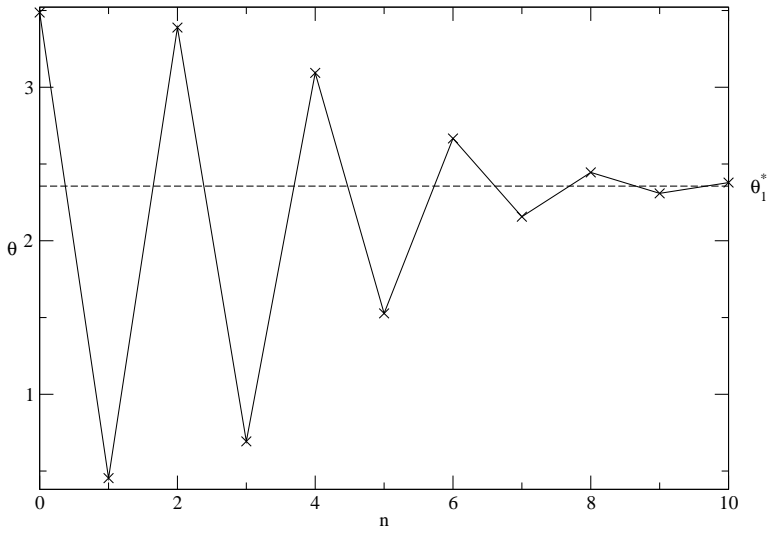

(d)

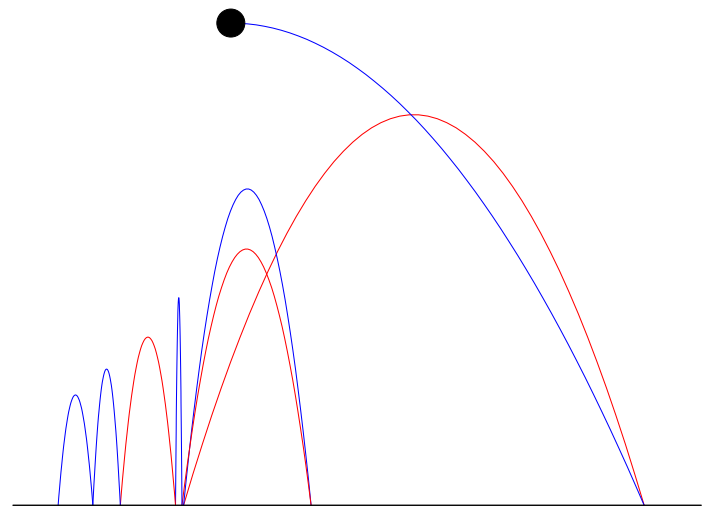

Figure 9: Results for $\frac{U_{0}}{\Omega_{0}}=0.36$. (a) Plot of $\theta_{n} \in\left[-\frac{\pi}{2}, \frac{\pi}{2}\right)$. (b) Plot of $\theta_{n} \in\left[\theta_{2}^{*}, \theta_{2}^{*}+\pi\right)$. (c) Plot of $V_{n}, U_{n}$ and $\Omega_{n}$. (d) The motion of the ball. A blue line indicates back spin $(\Omega>0)$ and a red line indicates forward spin $(\Omega<0)$. Half of the trajectory after the non-existent zeroth bounce is shown.

\section{A Ball Bouncing on Stairs}

Having considered in detail the problem of a ball bouncing on a flat horizontal surface, we now extend the analysis to the case of a piecewise-flat surface at two (or more) different levels, such as a staircase.

\subsection{The model}

Two flat horizontal surfaces occupy the half-planes $x>0, y=-a$ and $x<0, y=h-a$, where $h$ can be positive or negative. Thus, the centre of the ball when bouncing in $x>0$ is incident with the $x$-axis and the $y$-axis coincides with the step up or down in level. The half-planes are assumed to have the same tangential and normal coefficients of restitution, a restriction which is easily removed. Non-dimensional vertical distances can be defined by division by $v_{0}^{2} / g$ in a similar way to the horizontal distance $X$ in (21).

Consider the case of two bounces of the ball, where the first occurs at the level $y=-a$ and the second at $y=h-a$. We note that, since the horizontal velocity and spin are 
(a)

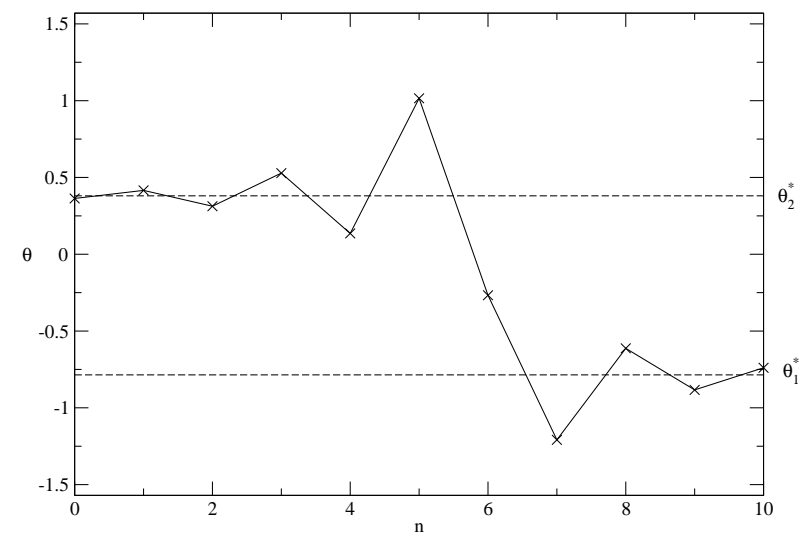

(c)

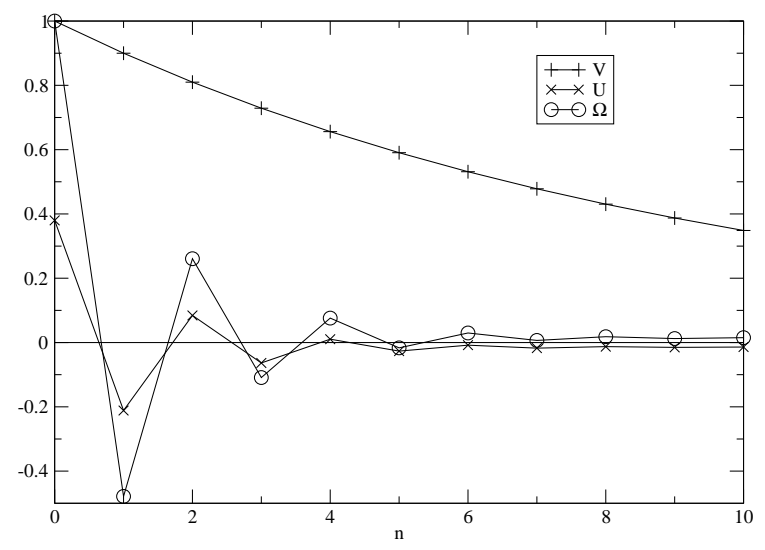

(b)

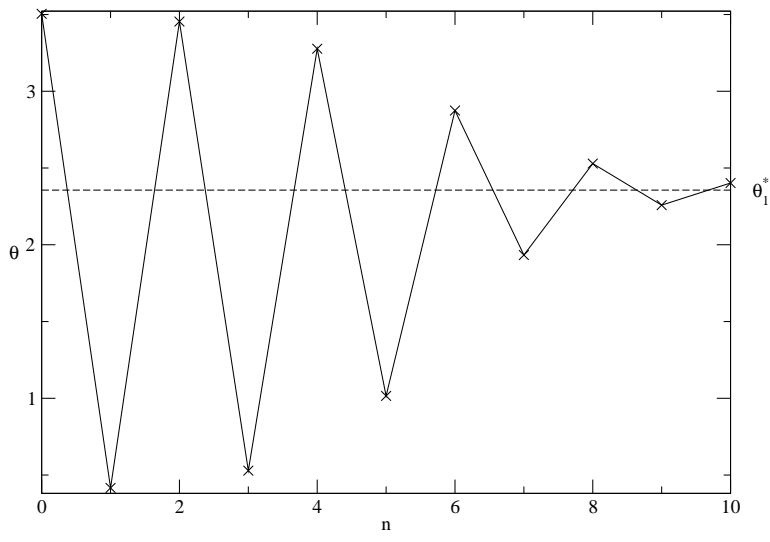

(d)

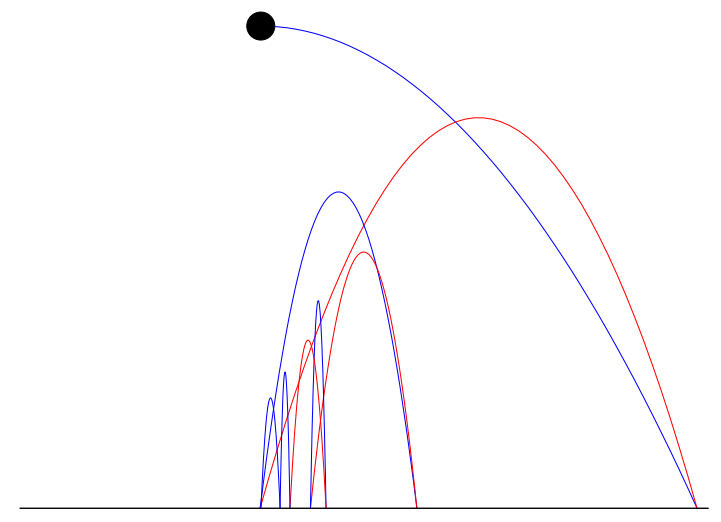

Figure 10: Results for $\frac{U_{0}}{\Omega_{0}}=0.38$. (a) Plot of $\theta_{n} \in\left[-\frac{\pi}{2}, \frac{\pi}{2}\right.$ ). (b) Plot of $\theta_{n} \in\left[\theta_{2}^{*}, \theta_{2}^{*}+\pi\right)$. (c) Plot of $V_{n}, U_{n}$ and $\Omega_{n}$. (d) The motion of the ball. A blue line indicates back spin $(\Omega>0)$ and a red line indicates forward spin $(\Omega<0)$. Half of the trajectory after the non-existent zeroth bounce is shown.

constant during the flight of the ball, (12) and (13) also apply in this case. Clearly there is a difference in the vertical direction and so there must be a change in equation (11).

Let $v_{n}$ be the vertical velocity immediately after the $n^{\text {th }}$ bounce at level $y=-a$ and let $v_{n+1}$ be the vertical velocity after the $(n+1)^{\text {th }}$ bounce at level $y=h-a$. Then

$$
v_{n+1}=-\beta v_{n+1}^{-}
$$

where $v_{n+1}^{-}<0$ is the vertical velocity immediately before the $(n+1)^{\text {th }}$ bounce. The latter follows from conservation of energy expressed (omitting contributions common to both sides) by the equation

$$
\frac{1}{2} m v_{n}^{2}=\frac{1}{2} m v_{n+1}^{-}{ }^{2}+m g h .
$$

Thus,

$$
v_{n+1}^{-}=-\sqrt{v_{n}^{2}-2 g h},
$$

and substituting this value into (53) gives

$$
v_{n+1}=\beta \sqrt{v_{n}^{2}-2 g h},
$$


a result which trivially requires that $v_{n}^{2}>2 g h$ (if this condition is not satisfied, the ball has insufficient energy to reach the step height). Dividing this equation by $v_{0}$, we obtain the corresponding non-dimensional equation

$$
V_{n+1}=\beta \sqrt{V_{n}^{2}-2 H}
$$

where $H=g h / v_{0}^{2}$ is a non-dimensional height parameter and (55) requires that $V_{n}^{2}>2 H$.

It is of interest to consider more closely the conditions for the ball to be able to mount the step after the $n^{\text {th }}$ bounce. Suppose that $h>0$ and that, at the $n^{\text {th }}$ bounce at the lower level, the centre of the ball is $\left(x_{n}, 0\right)$ with $x_{n}>a$. Then the equation of the ensuing trajectory of its centre is

$$
y=\frac{v_{n}}{u_{n}}\left(x-x_{n}\right)-\frac{g}{2 u_{n}^{2}}\left(x-x_{n}\right)^{2} .
$$

Define

$$
x_{n}^{ \pm}=-\frac{u_{n}}{g}\left\{v_{n} \pm \sqrt{v_{n}^{2}-2 g h}\right\}
$$

the two values of $x_{n}$ which make $y=h$ when $x=0$ in (56). In the situation envisaged $u_{n}$ is negative and hence, assuming that $v_{n}^{2}>2 g h, x_{n}^{ \pm}$are both positive. A necessary condition for the ball to mount the step is then $x_{n}^{-}<x_{n}<x_{n}^{+}$. However, this condition is not sufficient as the ball may still hit the corner of the step as it rises for some $x>0$.

To avoid such a collision, let $x_{n}^{-}+\delta$ be the abscissa of the centre of the ball when launched from the lower level in such a way that the parabolic trajectory touches the quarter circle

$$
x^{2}+(y-h+a)^{2}=a^{2}, \quad 0 \leq x \leq a, \quad h-a \leq y \leq h .
$$

The detailed calculation of $\delta$ in terms of the geometrical and launch data is difficult, involving a consideration of the character of the roots of the quartic equation resulting from the elimination of $y$ between (58) and (56) with $x_{n}$ replaced by $x_{n}^{-}+\delta$. It is not attempted here. However, in terms of $\delta$, necessary and sufficient conditions to have $y>h$ when $x=0$ are

$$
x_{n}^{-}+\delta<x_{n}<x_{n}^{+}
$$

together with $v_{n}^{2}>2 g h$. The left-hand inequality ensures that the ascending ball will not contact either the face or the edge of the step. With little loss of precision when $h \gg a,(59)$ can be replaced by the simpler sufficient condition

$$
x_{n}^{-}+a<x_{n}<x_{n}^{+} .
$$

The non-dimensionalised form of (59) is

$$
X_{n}^{-}+\Delta<X_{n}<X_{n}^{+} \text {and } V_{n}^{2}>2 H
$$

where

$$
X_{n}^{ \pm}=-U_{n}\left\{V_{n} \pm \sqrt{V_{n}^{2}-2 H}\right\}, \quad \Delta=\frac{g \delta}{v_{0}^{2}} .
$$

When the ball bounces from the higher step with $x_{n}<0$ and $u_{n}>0$, in order to land on the lower step the range corresponding to (60) is $a-2 u_{n} v_{n} / g<x_{n}<0$, clearly independent of $h$. In all subsequent analysis it is assumed that conditions such as (61) are satisfied, that is, we reject situations in which the ball strikes either the face or the edge of the step. 
The horizontal displacement between bounces at the two different heights in the lower step to higher step case also follows from (56) by setting $x=x_{n+1}$ when $y=h$. Since $u_{n}<0$, the appropriate negative root of the quadratic equation for $x_{n+1}-x_{n}$ is

$$
x_{n+1}-x_{n}=\frac{u_{n}}{g}\left\{v_{n}+\sqrt{v_{n}^{2}-2 g h}\right\} \text {. }
$$

In non-dimensional coordinates, this becomes

$$
X_{n+1}-X_{n}=U_{n}\left(V_{n}+\sqrt{V_{n}^{2}-2 H}\right)
$$

It is easily verified that this result also applies for a bounce from the higher to the lower step when $X_{n}<0$ and $U_{n}>0$ provided that $H$ is replaced by $-H$.

\subsection{Oscillating bounces at different heights}

In Sections 4 and 5 we considered in detail the conditions required for direction and spin reversals of a ball. Since the equations governing the dynamics of $U$ and $\Omega$ are the same when the bounces occur at different heights, all the results in these sections apply in this case also. Thus, we now consider the case where $U_{0} / \Omega_{0}$ is chosen so that there are at least $N$ reversals of direction of the ball. We choose the profile of the floor as in the previous section with $h>0$, and suppose that the motion starts with the ball leaving floor level in $x>a$, with linear and angular velocity components $u_{0}, v_{0}$ and $\omega_{0}$, all of which are non-dimensionalised in the usual way on division by $v_{0}$ or $v_{0} / a$ as appropriate. We treat the initial projection situation as the result of a zeroth bounce in $x>a$; thus even numbered bounces take place on $y=-a$ and odd numbered bounces on $y=h-a$. Denoting by $x_{n}$ the abscissa of the ball centre at the $n^{\text {th }}$ bounce, we assume that $x_{0}>a$ is chosen so that $x_{2 n-1}<0$ and $x_{2 n}>a$ for at least $N$ bounces.

With this scenario, the non-dimensionalised dynamics in the vertical direction is given by

$$
V_{n+1}=\beta \sqrt{V_{n}^{2}-2 H}
$$

at even numbered bounces and by

$$
V_{n+1}=\beta \sqrt{V_{n}^{2}+2 H}
$$

at odd numbered bounces. Note that the sign of the $2 H$ - term changes in this case since the odd numbered bounces involve the ball moving to a level $H$ lower than the current bounce. Equations (63) and (64) can be combined into the single equation

$$
V_{n+1}=\beta \sqrt{V_{n}^{2}-2(-1)^{n} H}
$$

for $n=0,1, \ldots, N$. Squaring gives

$$
V_{n+1}^{2}=\beta^{2}\left\{V_{n}^{2}-2(-1)^{n} H\right\}, \quad n=0,1, \ldots, N,
$$

a difference equation whose solution, subject to $V_{0}=1$, is

$$
V_{n}^{2}=\beta^{2 n}+2(-1)^{n} \beta^{2} H\left(\frac{1-\left(-\beta^{2}\right)^{n}}{1+\beta^{2}}\right) .
$$


As noted in the previous section and from (63), for the ball to reach the higher level the even bounces must satisfy

$$
V_{2 n}^{2}>2 H .
$$

Substituting for $V_{2 n}$ from (66) requires that

$$
\beta^{4 n}+2 \beta^{2} H\left(\frac{1-\beta^{4 n}}{1+\beta^{2}}\right)>2 H,
$$

that is

$$
H<\frac{\beta^{4 n}\left(1+\beta^{2}\right)}{2\left(1+\beta^{4 n+2}\right)} .
$$

The quantity on the right-hand side of (67) is the value of $H$ which ensures that the maximum height reached on the $2 n^{\text {th }}$ bounce is precisely $H$. Converting back to the original coordinates and rearranging, (67) becomes

$$
v_{0}^{2}>\frac{2 g h\left(1+\beta^{4 n+2}\right)}{\beta^{4 n}\left(1+\beta^{2}\right)},
$$

which provides a lower bound on the initial vertical velocity $v_{0}$ to ensure that the $2 n^{\text {th }}$ bounce does give the ball sufficient height to reach the raised step.

\subsection{Bouncing down a staircase}

The bouncing superball can have two types of motion, namely oscillating backwards and forwards or moving in the same direction at each bounce. In the previous section we treated the case of oscillating motion with a single step. We now consider the case of bounces which all occur in the same direction and combine this with a full staircase to see what behaviour is possible for the ball bouncing down the stairs.

All the stairs in the staircase are of equal height $h$, which, in nondimensional coordinates, corresponds to $H=g h / v_{0}^{2}$. Assuming that the ball bounces one stair lower at each bounce, the dynamics in the vertical direction is described by (55) with $H$ replaced by $-H$ and is therefore given by

$$
V_{n+1}=\beta \sqrt{V_{n}^{2}+2 H}=f\left(V_{n}\right), \text { say. }
$$

Squaring (68), the solution, subject to $V_{0}=1$, is found as

$$
V_{n}^{2}=\beta^{2 n}+2 H \beta^{2}\left(\frac{1-\beta^{2 n}}{1-\beta^{2}}\right) .
$$

Clearly,

$$
V^{*}=\lim _{n \rightarrow \infty} V_{n}=\beta \sqrt{\frac{2 H}{1-\beta^{2}}},
$$

where $V^{*}$ is the fixed point of the map (68). Further, it is stable since $f^{\prime}\left(V^{*}\right)=\beta^{2}<1$. It also follows from (69) and (70) that

$$
V_{n+1}^{2}-V_{n}^{2}=\beta^{2 n}\left(\left(V^{*}\right)^{2}-1\right) .
$$

Thus, for all $n, V_{n+1}<V_{n}$ if $V^{*}<1$ and $V_{n+1}>V_{n}$ if $V^{*}>1$. This implies that the height the ball bounces from each stair as the vertical velocity approaches its limiting value of $V^{*}$ will be successively either higher or lower than the previous bounce, depending on whether 
$V^{*}>1\left(=V_{0}\right)$ or $V^{*}<1$. In dimensional variables, the conditions $V^{*}>1$ and $V^{*}<1$ are equivalent to $v_{0}<v^{*}$ and $v_{0}>v^{*}$, where

$$
v^{*}=\beta \sqrt{\frac{2 g h}{1-\beta^{2}}} .
$$

We know from (20) that the ratio $U_{n} / \Omega_{n}$ tends to the stable fixed point -1 as $n \rightarrow \infty$, provided that $U_{0} / \Omega_{0} \neq 2 / 5$. Since this is associated with the eigenmode with corresponding eigenvalue $\lambda_{1}=1$, it follows that this fixed point also gives $U_{n+1}=U_{n}$ and $\Omega_{n+1}=\Omega_{n}$. Thus, the ball bouncing down the stairs will tend towards the combined stable state of $V=V^{*}$ and $U / \Omega=-1$ as $n \rightarrow \infty$ and in this state, the bounce from each stair is identical.

In terms of the horizontal displacement between bounces, we replace $H$ by $-H$ in (62) to give

$$
X_{n+1}-X_{n}=U_{n}\left(V_{n}+\sqrt{V_{n}^{2}+2 H}\right),
$$

where, as before, $X_{n}$ is the dimensionless horizontal coordinate of the $n^{\text {th }}$ bounce. At the stable fixed point of the iteration, $V_{n}=V^{*}$, where $V^{*}$ is given by (70), and $U_{n}=U_{0}$ for all $n$. In this case, the horizontal distance travelled between bounces is constant, and is given by

$$
\begin{aligned}
X_{n+1}-X_{n} & =U_{0}\left(V^{*}+\sqrt{\left(V^{*}\right)^{2}+2 H}\right) \\
& =(1+\beta) U_{0} \sqrt{\frac{2 H}{1-\beta^{2}}} .
\end{aligned}
$$

Suppose that the non-dimensional tread-width of each stair is $W$; then the ball will bounce at the same point on each stair if $U_{0}$ is chosen so that

$$
W=(1+\beta) U_{0} \sqrt{\frac{2 H}{1-\beta^{2}}},
$$

that is

$$
U_{0}=\frac{W}{1+\beta} \sqrt{\frac{1-\beta^{2}}{2 H}} .
$$

\subsection{Examples}

We again take the coefficients of restitution as $\beta=0.9$ and $\alpha=0.5$. For oscillating motion up and down a step, we take $U_{0}=0.45$ and $\Omega_{0}=1$, which results in four direction reversals and five spin reversals, and choose the height of the step to be $H=0.1$. The corresponding motion is then shown in Fig. 11.

To simulate the ball bouncing down the stairs, we select initial conditions with $\Omega_{0}=-U_{0}$, corresponding to the rolling eigenmode. We choose $U_{0}$ according to (71) with the width of each stair as $W=1$, giving $U_{0}=0.362738$. We also choose the height of the stairs to be $H=0.2$. With these values, we find from (70) that $V^{*}=1.305857$ and, since $V_{0}<V^{*}$, $V_{n}$ will monotonically increase towards this limiting value as $n \rightarrow \infty$. The resulting motion is shown in Fig. 12. Note that the position of the bounce on each stair gradually moves towards the riser, and will tend to a limiting value as the motion approaches its fixed-point values.

Many other forms of motion are possible on a staircase. One such example is shown in Fig. 13, where the initial conditions are $U_{0}=0.3$ and $\Omega_{0}=0.681818$, giving $U_{0} / \Omega_{0}=0.44$ which again corresponds to four direction reversals. 


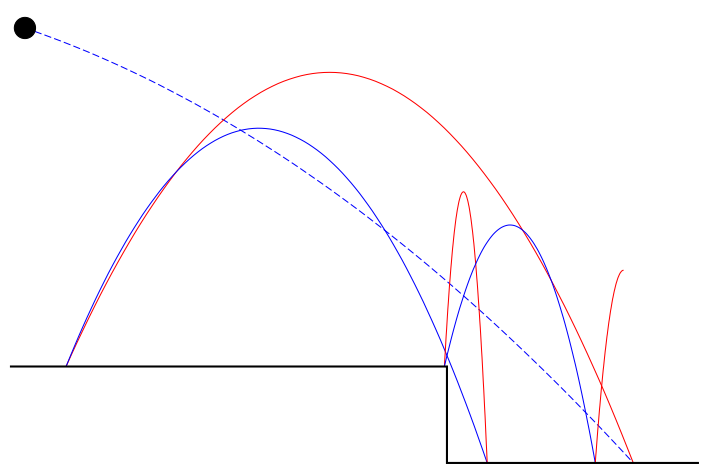

Figure 11: Oscillating motion of a ball up and down a step. A blue line indicates back spin $(\Omega>0)$ and a red line indicates forward spin $(\Omega<0)$. The dashed line shows a trajectory that gives the required initial conditions after the zeroth bounce.

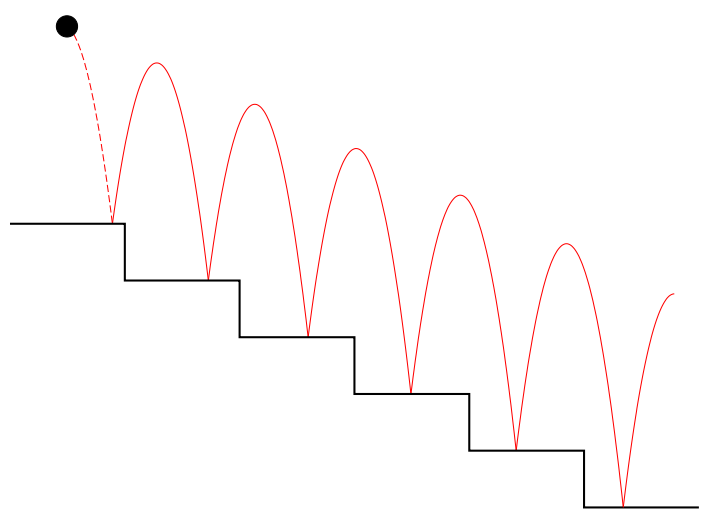

Figure 12: A ball bouncing down a staircase. The red lines indicate that the ball has forward spin $(\Omega>0)$. The dashed line shows a trajectory that gives the required initial conditions after the zeroth bounce.

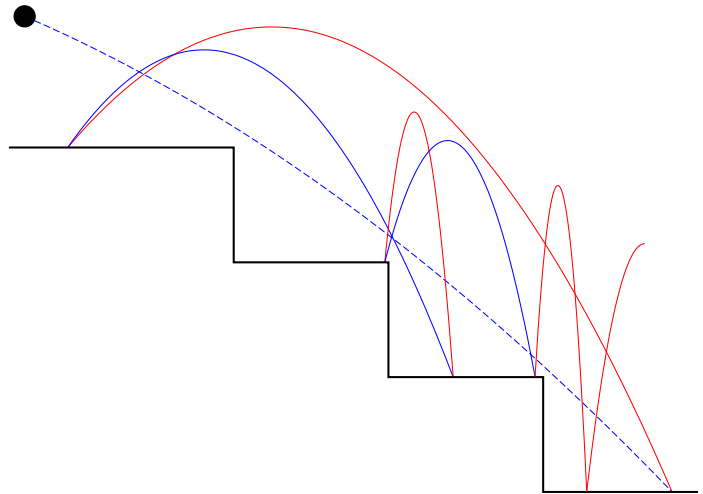

Figure 13: Oscillating motion of a ball on a staircase. A blue line indicates back spin $(\Omega>0)$ and a red line indicates forward spin $(\Omega<0)$. The dashed line shows a trajectory that gives the required initial conditions after the zeroth bounce. 


\section{References}

[1] Bollt, E.M. and Klebanoff, A., A new and simple chaos toy. Int. J. Bif. Chaos 12, 1843-1857, 2002.

[2] Bridges, R., The spin of a bouncing 'superball'. Phys. Educ. 26, 350-354, 1991.

[3] Caughey, T.K., A mathematical model of the rattleback. Int. J. Nonlin. Mech. 15, 293-302, 1980.

[4] Cross, R., The bounce of a ball. Am. J. Phys. 67, 222-227, 1999.

[5] Cross, R., Measurements of the horizontal coefficient of restitution for a superball and a tennis ball. Am. J. Phys. 70, 482-489, 2002.

[6] Cross, R., Grip-slip behavior of a bouncing ball. Am. J. Phys. 70, 1093-1102, 2002.

[7] Doménech, A., A classical experiment revisited: The bounce of balls and superballs in three dimensions. Am. J. Phys. 73, 28-36, 2005.

[8] Garcia A and Hubbard, M., Spin reversal of the rattleback - theory and experiment. Proc. Roy. Soc. Lond. Ser. A 418 (1854), 88-92, 1988.

[9] Garwin, R., Kinematics of an ultraelastic rough ball. Am. J. Phys. 37, 88-92, 1969.

[10] Hefner, B.T., The kinematics of a superball bouncing between two vertical surfaces. Am. J. Phys. 72, 875-883, 2004.

[11] Kane, T.R. and Levinson, D.A., Realistic mathematical modeling of the rattleback. Int. J Nonlin. Mech. 17, 175-186, 1982.

[12] Marsden, J.E. and Romero, L.A., Tippe top inversion as a dissipation-induced instability. SIAM J. Appl. Dyn. Syst. 3, 352-377, 2004.

[13] Or, A.C., The dynamics of a Tippe top. SIAM J. Appl. Math. 54, 597-609, 1994.

[14] Routh, E.J., An Elementary Treatise on the Dynamics of a System of Rigid Bodies, 6th edition, MacMillan, London, 1897. 